\title{
Article \\ Applying Parallel and Distributed Models on Bio-Inspired Algorithms via a Clustering Method
}

\author{
Álvaro Gómez-Rubio ${ }^{1}$, Ricardo Soto ${ }^{1}{ }^{\circledR}$, Broderick Crawford $^{1}{ }^{\circledR}$, Adrián Jaramillo ${ }^{1}$, David Mancilla ${ }^{2}$, \\ Carlos Castro $^{3}(\mathbb{1})$ and Rodrigo Olivares ${ }^{4, *}$ (i)
}

1 Escuela de Ingeniería Informática, Pontificia Universidad Católica de Valparaíso, Valparaíso 2362807, Chile; alvaro.gomez.r@mail.pucv.cl (Á.G.-R.); ricardo.soto@pucv.cl (R.S.); broderick.crawford@pucv.cl (B.C.); adrian.jaramillo.s@mail.pucv.cl (A.J.)

2 Independent Researcher, Valparaíso 2362807, Chile; david.mancilla@axity.com

3 Departamento de Informática, Universidad Técnica Federico Santa María, Valparaíso 2390123, Chile; carlos.castro@inf.utfsm.cl

4 Escuela de Ingeniería Informática, Universidad de Valparaíso, Valparaíso 2362905, Chile

* Correspondence: rodrigo.olivares@uv.cl

check for

updates

Citation: Gómez-Rubio, Á.; Soto, R.; Crawford, B.; Jaramillo, A.; Mancilla, D.; Castro, C.; Olivares, R. Applying Parallel and Distributed Models on Bio-Inspired Algorithms via a Clustering Method. Mathematics 2022, 10, 274. https://doi.org/10.3390/ math10020274

Academic Editors: Alfredo Milani and Alessandro Niccolai

Received: 4 December 2021

Accepted: 10 January 2022

Published: 16 January 2022

Publisher's Note: MDPI stays neutral with regard to jurisdictional claims in published maps and institutional affiliations.

Copyright: (C) 2022 by the authors. Licensee MDPI, Basel, Switzerland. This article is an open access article distributed under the terms and conditions of the Creative Commons Attribution (CC BY) license (https:// creativecommons.org/licenses/by/ $4.0 /)$.

\begin{abstract}
In the world of optimization, especially concerning metaheuristics, solving complex problems represented by applying big data and constraint instances can be difficult. This is mainly due to the difficulty of implementing efficient solutions that can solve complex optimization problems in adequate time, which do exist in different industries. Big data has demonstrated its efficiency in solving different concerns in information management. In this paper, an approach based on multiprocessing is proposed wherein clusterization and parallelism are used together to improve the search process of metaheuristics when solving large instances of complex optimization problems, incorporating collaborative elements that enhance the quality of the solution. The proposal deals with machine learning algorithms to improve the segmentation of the search space. Particularly, two different clustering methods belonging to automatic learning techniques, are implemented on bio-inspired algorithms to smartly initialize their solution population, and then organize the resolution from the beginning of the search. The results show that this approach is competitive with other techniques in solving a large set of cases of a well-known NP-hard problem without incorporating too much additional complexity into the metaheuristic algorithms.
\end{abstract}

Keywords: distributed metaheuristics; parallel metaheuristic; big data clustering; optimization problems

\section{Introduction}

In the optimization sphere, the solving of distinctly large and complex problems also involves important information management issues [1]. For instance, most of these problems are currently tackled using metaheuristic algorithms, which attempt to optimize the values obtained by an objective function; this is done through an exploration of a large number of potential solutions, carried out by a population of agents [2]. This exploration is governed by a movement/disturbance operator, which decides the regions to be analyzed. Naturally, this process involves huge areas of information that often require large computational efforts to be analyzed and may not deliver acceptable results [3]. Thus, there is a problem to be solved in the exploration/exploitation duality to concentrate the search on the areas known as the "neighborhoods of solutions", where there is a higher probability of finding better quality solutions [3]. The objective of this study is to present a framework to improve the efficiency and effectiveness of the metaheuristic search process by increasing the number of global optima found and reducing the time or computational costs taken for the same. This is done by implementing the search process in several runs of metaheuristic algorithms, in an autonomous and parallel way (nodes). However, this is 
to be coordinated and with the grouping of the solution space in groups of solutions with similar characteristics by means of machine learning algorithms' methods for clustering. These algorithms are used at the beginning and during the search process to analyze the available solutions according to various criteria and cluster them in terms of similarity. The framework uses the best solutions found for each cluster from all nodes and distributes them to replace the lowest quality solutions available at that time. In addition, parallel execution and exploitation of these segregated spaces are performed by distributing the searches through parallelism based on big data tools.

Thus, this research aims to demonstrate that the subdivision of the solution space into homogeneous groups, combined with the cooperation between the nodes generated according to the results found with the proposed framework, versus those by the standard versions of metaheuristics, allows for the improvement of the exploration and exploitation characteristics independent of the algorithms used in the process. Additionally, the use of a distributed platform enables cooperation during runtime and decreases the waiting time to obtain an optimal solution, compared to the time required by non-distributed algorithms. In a distributed platform, the total computation time cannot be measured akin to a standalone execution, so the total number of evaluations of the objective function has also been used as an indicator (MFE).

We have implemented an instance of this framework using four different population metaheuristic algorithms - the binary black hole (BBH) [4], the soccer league competition (SLC) [5], the shuffled frog-leaping algorithm (SFLA) [6] and the spotted hyena optimizer (SHO) [7] to combine the different search strategies that each one presents. These algorithms were selected because they are population-based, and there are previous work on tuned versions, which facilitated further comparisons. However, the architecture can be extended to almost any iterative population metaheuristics.

The rest of this paper is organized as follows: Section 2 explains the related work concerning the combination of metaheuristics and machine learning algorithms. Section 3 details the proposed framework, and Section 4 reviews the metaheuristics used and the addressed optimization problem. The methodology is demonstrated in Section 5, the statistical analysis used is presented in Section 6, and the experimental results are detailed in Section 7. Finally, the conclusions are presented in Section 8.

\section{Related Work}

The relationship of metaheuristics to data science is broad and mainly framed as two primary types of collaboration $[8,9]$. On the one hand, metaheuristics can be a powerful auxiliary tool for different machine learning algorithms that need to solve NP-hard problems, or require fast optimization for large volumes of data with complex constraints, such as automatically finding the optimal value of $\mathrm{K}$ for the Kmeans clustering algorithm [10], finding tuning parameters by searching for optimal values [11], or problems that require minimization or maximization for classification algorithms to improve their efficiency [12-14]. These types of situations are common in tasks associated with image recognition, pattern recognition or deep learning $[15,16]$.

The second approach corresponds to the opposite strategy, i.e., the combination of both techniques in a common and unique process to solve complex optimization or other types of problems. Metaheuristics, being a non-deterministic algorithm, must face a series of unpredictable situations in the search for solutions, and it is at this point where machine learning algorithms support them. Oliveira et al. [17] proposed, for example, a framework that enhances clustering by combining Kmeans with metaheuristics in a distributed environment and Zhang et al. proposed a hybrid solution for geotechnical problems [18]. On the other hand, machine learning and metaheuristics have been combined to solve problems, such as disastrous natural hazards [19], classification of biomedical data [20] and multiple other applications [21].

In optimization algorithms, and especially in metaheuristics, there is currently a promising line of research called "hybridization," which consists of combining metaheuris- 
tic algorithms with various other types of approaches, mainly in the area of machine learning. This is no longer an assistive approach, but involves the use of both techniques together to solve problems that are too complex to be solved by each of them separately [22,23]. New computational capabilities have brought new problems that require special approaches to be solved, such as massive image processing, and it is in this context that hybrid algorithms are presented as effective solutions [24].

This last approach has shown improved results for complex problems that require high computational consumption and complex parameter tuning. This is because the combination of techniques allows the automation of many of the tasks that previously had to be performed manually [25]. Among the areas in which effective solutions have been implemented are healthcare [26], transportation [27] and energy [28]. Usually, the implementation of parallel metaheuristics is done by means of complex programming techniques and extensive use of available hardware [29]. This results in high implementation complexity, platform dependency and extensive alteration of the algorithms used [30].

\section{Preliminaries}

\subsection{Framework Description}

A relevant aspect in optimization using metaheuristics is the initial generation of solutions for the search space, which is generally a random process that validates that only feasible solutions are generated. In traditional implementations of metaheuristics, this aspect is usually adjusted by tuning the specific method that creates the initial set of solutions. However, in distributed environments, additional aspects must be taken into consideration. On the other hand, during the search process of a population metaheuristic, the set of initial solutions change iteratively, as the algorithm converges towards neighborhoods and solutions of better quality, which is always a stochastic process and regulated by the operators that each algorithm has [31].

In the present work, the above mentioned strategy is implemented to incorporate some new elements. First, the search is not performed by a single instance of a metaheuristic algorithm, but rather a series of distributed executions are generated, each separately but in communication with a central controller component. Each node corresponds to a particular algorithm execution, so it is necessary to segment the search space in order to distribute it among the different nodes and stimulate exploration. Two aspects to be performed at the beginning of the process are the determination of the search space in terms of partitioning criteria, and the number of nodes that will be needed later on. After multiple exploratory experiments, two criteria were defined to differentiate groups of solutions: the quality of fitness and the distance from a randomly generated vector at the beginning of the process. These two elements together allow a good differentiation among solution groups from a primeval set.

As mentioned above, a strategy has been established to enable collaboration levels between nodes and thus allow better results to be shared among different executions and nodes. The different nodes are triggered and coordinated by a central coordinator (CC), which iteratively receives the best found fitness for each performance-basically, a parameter set interval of iterations, in such a way that each node reports its best fitness to the CC and finds the best ones from all. Once determined, the CC distributes the selected best solutions toward all nodes, thus improving the quality of all searching spaces. The mechanism for the interaction between nodes is based on the periodic supervision of the results of each instance by the CC, which, in the event of a significant improvement in any of them, distributes part of the set of solutions with better fitness to the others. To achieve this, the algorithm's executions are interrupted periodically after a certain number of iterations, according to a global parameter, to inform the CC of their results and the reception of a subset of better quality solutions found by another node, which contributes to enriching its own. The value of this parameter was experimentally established in 100 iterations, which has been repeated throughout the search process for all nodes. Once one of the nodes finds 
the global optimum or the exit criterion, the CC reviews the solutions found and delivers the found optimum, along with some other values. The Figure 1 shows this relationship:

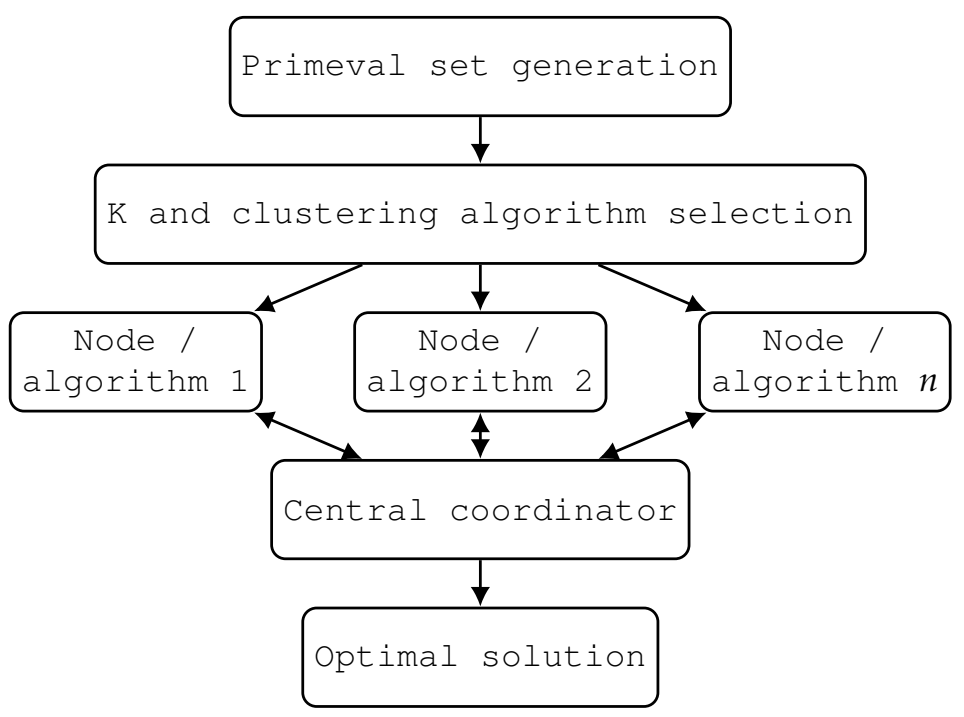

Figure 1. Collaboration strategy.

Upon analysis of the figure from top to bottom, the generation of an extended set of feasible solutions called a "primeval set" is observed. The process continues by determining the clustering algorithm to use and the appropriate number of clusters to generate (K), as can see in the second stage. In general, different clustering algorithms can deliver different results for the same data [32]. Therefore, to ensure an adequate distribution of solutions, two data science algorithms were used, which were then compared: Kmeans [33] and agglomerative clustering [34]. This stage was only executed once centrally, requiring a minimum portion of time, with respect to the total time taken by the entire process. At runtime, it was determined which of the two methods is better balanced and distributed for different quantities of groups or clusters that are then generated in the third step. These clusters were then used as input to a MapReduce approach, making it responsible for parallelizing the search through different nodes. It was determined that at least the same number of nodes will be generated as clusters; thus, the number of nodes will always be greater than K. Each node executes a metaheuristic with its assigned group of solutions, while a central layer coordinates its operation. The solid red line represents the interactions of nodes with the CC every 100 iterations to report the results, and the dotted blue line represents the distribution of values selected by the CC. The use of parallel populations makes it possible to multiply the processing capacity, achieving optimal results in less time compared to a standalone architecture. Additionally, the distributed execution offers the possibility of dividing the search into different neighborhoods of solutions, increasing the exploration/exploitation capacity within the search process.

Best solutions are exchanged between nodes permanently over a set number of iterations. In the event that one of the nodes finds the global optimum, it will inform the $\mathrm{CC}$ at the end of the current set of iterations and the cluster runs will stop. On the other hand, those solutions closer to the global optimum will prevail as the iterations progress, since they will be distributed among the nodes and replace those with worse fitness. Then, the very mechanics of permanent exchange of better quality solutions causes that always the best fitness solutions found are present in all the nodes. Considering that the best solutions found replace the worst ones of the other nodes, the solutions of neighborhoods that so far are of regular or acceptable quality will be preserved, in order to stimulate the exploration in other neighborhoods while minimizing the stagnation in local solutions.

For each algorithm used, their respective parameters were determined experimentally by means of their standalone execution. The same values were then used in the distributed executions, in order to make possible the comparison of results between both modalities. 
We have illustrated notable improvements over a large instance set of the well-known NP-hard set covering problem, where the proposed approach is able to compete with other modern metaheuristics, while clearly outperforming the non-parallel version of some algorithms. Moreover, the framework presented is flexible enough to allow the addition of new algorithms, or replacing those used in this work. This is relevant, as the results show heterogeneous performance among the different algorithms.

\subsection{Clustering}

To separate the solutions into different groups, according to the described characteristics, the Kmeans [33] and agglomerative clustering [34] algorithms were used. The used clustering algorithms used are learning techniques based on model-free strategies, which do not require a training phase and can therefore be applied without the need for a prior representative sample of solutions for each algorithm. Both algorithms are commonly used unsupervised machine learning techniques know for being flexible, quick and reliable [35].

To use them, it is crucial to previously determine a value that corresponds to the number of clusters into which data will be divided. This number is represented by " $\mathrm{K}$ ". Both algorithms are executed, and their results are compared using the silhouette coefficient method [36] to determine the appropriate $\mathrm{K}$ value, and the algorithm that delivers the better results. Once the value of $\mathrm{K}$ is determined, the selected algorithm is applied to the entire initial set of solutions, assigning a cluster number to each of them.

Subsequently, the solutions are separated and grouped according to the assigned cluster, extracting the solutions needed to cover each one according to the initial parameter of each algorithm, similar to selecting the solutions of best fitness for each cluster. At this point, the distributed execution can start. The $\mathrm{K}$ nodes are generated and assigned one of the predefined algorithms: BBH, SLC, SFLA and SHO. If K is greater than the number of available algorithms (four in this research), the algorithms are repeated in a fixed sequence until the defined $\mathrm{K}$ is completed. The number of algorithms used is arbitrary and responds to tactical reasons, and there is no reason for there to be more of them.

\section{Implemented Metaheuristics and Optimization Problem}

For experimental purposes, we decided to tackle a well-known optimization problem to compare our results with previous results and have an abundant bibliography. For this reason, a classical NP-hard problem, the set covering problem (SCP), was selected. However, the proposed framework can be easily adapted to solve some other more complex modeling problems.

Regarding the algorithms used, four factors were considered: First, population algorithms were selected because they are more directly adaptable to the proposed architecture, although other types of algorithms can also be easily incorporated. Second, relatively modern algorithms with reported results were preferred in order to have a first-hand approximation of their effectiveness, and the recommended parameter values for each of them. Third, they were algorithms with differences in their convergence rates to determine if the variable is a relevant factor in the values obtained. Finally, considering that what we want to evaluate is the difference in behavior as a whole, and not the intrinsic nature and quality of each of them, we chose some that had already been used by researchers in the past, in order to facilitate their adaptation.

\subsection{Set Covering Problem}

Considering $\left(a_{i j}\right)$ an binary array A defined by $m$ rows and $n$ columns, and a $C$ vector $\left(c_{j}\right)$ of $n$ columns containing the costs assigned to each other, then we can then define the SCP as follows:

$$
\operatorname{Minimize} \sum_{j=1}^{n} c_{j} x_{j}
$$

where: 


$$
\begin{gathered}
\sum_{j=1}^{n} a_{i j} x_{j} \geq 1 \forall i \in\{1, \ldots, m\} \\
x_{j} \in\{0,1\} ; j \in\{1, \ldots, n\}
\end{gathered}
$$

This ensures that each row is covered by at least one column where there is a cost associated with it. This problem was introduced in 1972 by Karp [37] and was used to optimize problems of element location that provide spatial coverage, such as community services [38], telecommunications antennas [39] and others [40].

\subsection{Metaheuristics Implemented}

The algorithms used in this work were selected based on two aspects. Firstly, they correspond to population algorithms of different complexities, from a very simple one such as BBH to others with more complex operators, such as SFLA and SHO. Secondly, the research team has several published works using these same algorithms, so the work of adaptation, tuning and comparison of results was relatively easy. Notwithstanding these observations, the work focuses on the collaborative and distributed execution of the algorithms and not on the nature, operators or parametrization of them, so that the proposed framework can be extended to many other algorithms, traditional or new.

\subsubsection{Binary Black Hole Algorithm}

The binary black hole algorithm [4] faces the problem of determining solutions through the development of a set of stars called universe, using a population algorithm. It proposes the rotation of the universe around the star that has the best fitness, i.e., the one with lowest fitness value. This rotation is applied by an operator that moves each star in each iteration of the algorithm, and determines if there is a new black hole in each cycle. If it so happens, it replaces the previous one. This operation is repeated until the stop criterion is met, making the last black hole found the proposed final solution. Eventually, a star may exceed the distance defined by the radius of the event horizon. In this case, the star can collapse into a black hole and will be removed from the universe, substituted by a new star. The input for the algorithm are the universe size and max iterations, and the output is the last black hole found. The procedure can be seen in Algorithm 1.

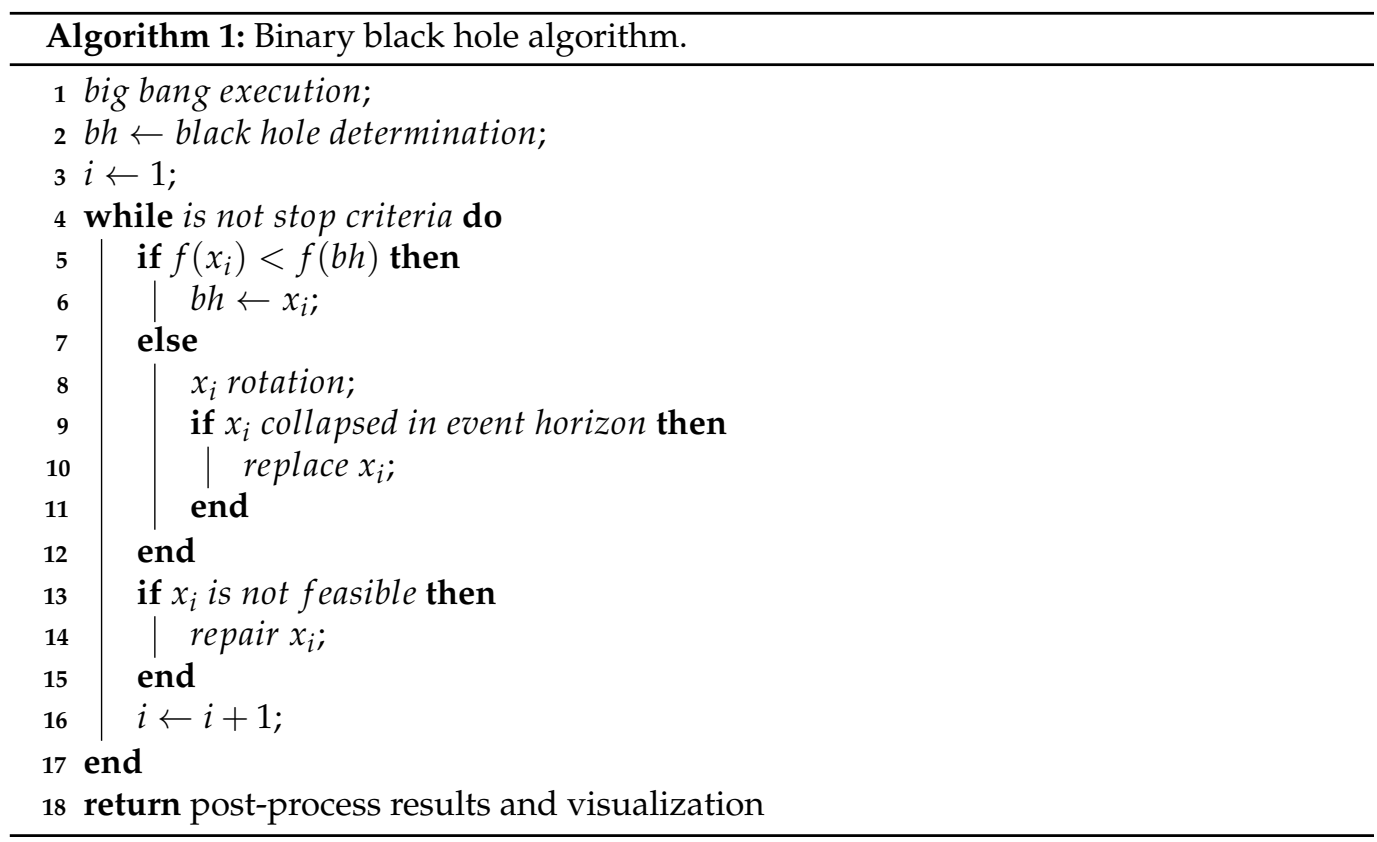




\subsubsection{Soccer League Competition Algorithm}

Soccer league competition algorithm is a metaheuristic model based on soccer competitions [41]. This approach considers solutions as soccer players, and sets them up as soccer teams. There are movement operators applied to the players, emulating the dynamics generated in real matches to win and become the best football player of the season. Players are defined as two types: fixed players and substitutes ones. The convergence of the population towards the global optimum is achieved through competition among teams competing for the top positions in the classification table. Players also compete to be fixed and be the star ones of their respective teams. The goal is to find the best feasible solution that achieves the best value from the evaluation function, using operators who evaluate the players and teams' performances and exchange solutions. The algorithm inputs are the number of teams and fixed and substitute players. The output is the star player. Algorithm 2 details the resolution process.

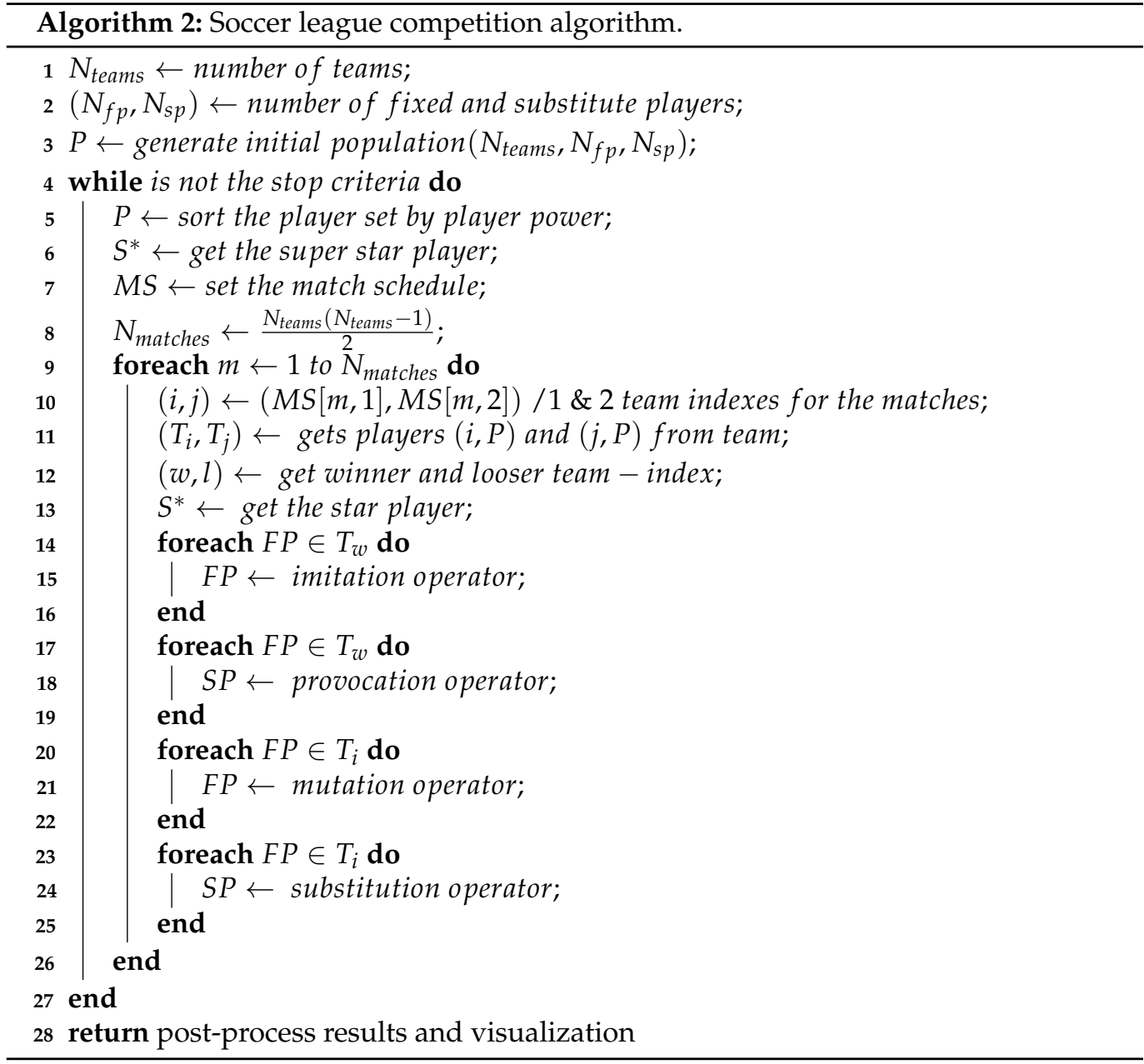

\subsubsection{Shuffled Frog-Leaping Algorithm}

The shuffled frog leaping algorithm is a population-based cooperative search metaheuristic, inspired by natural memetics [6]. The algorithm manages a set of interacting virtual population of frogs, partitioned into different memeplexes, including elements of local search and global information exchange. The frogs simulate hosts of memes, where a meme is a basic unit of cultural evolution. This algorithm performs independent local searches on each memeplex, using a variant of the particle swarm optimization method. The frogs are shuffled periodically and reorganized into new memeplexes. This technique is similar to that used in the complex evolutionary shuffle algorithm, to ensure global exploration. To improve randomness, frogs are randomly generated and replaced in the 
population. The algorithm input are the number of memeplexes, frogs in each memeplex, iterations in each memeplex and maximum algorithm iterations, while the output is the best frog fitness. Algorithm 3 explains the step-by-step of the resolution process.

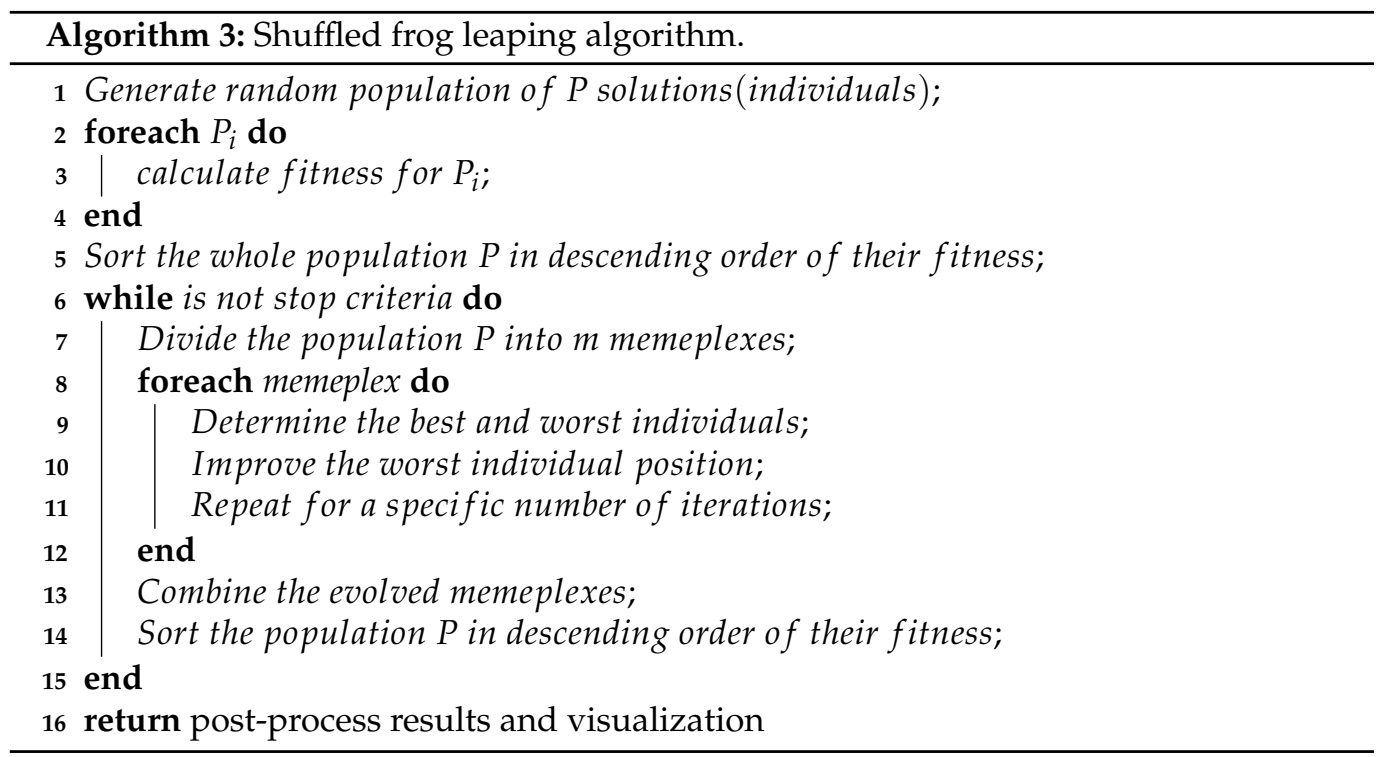

\subsubsection{Spotted Hyena Optimizer Algorithm}

The fundamental concept of this algorithm is the natural hunting strategy of the spotted hyena [7]. The three basic steps of this proposed spotted hyena optimizer algorithm are searching, encircling, and attacking for prey. Spotted hyenas first search for prey, depending on their position residing in the solution space, and then move away from each other to attack their prey.

Similarly, we use operators with random values to force the search agents to move far away from the prey, thereby stimulating the $\mathrm{SHO}$ algorithm to search globally. To encircle the prey, the best solution is considered the target prey, and the other search agents or spotted hyenas can update their positions according to this optimal solution. The other search agents will try to update their positions after the best search candidate solution is defined. The third step of SHO algorithm is the attack strategy, which sets up a cluster of optimal solutions against the best search agent, and updates the positions of other search agents. The input for algorithm is the population $P_{i}(i=1,2, \ldots, n)$ and the output is the best search agent. Algorithm 4 presents the search process. 


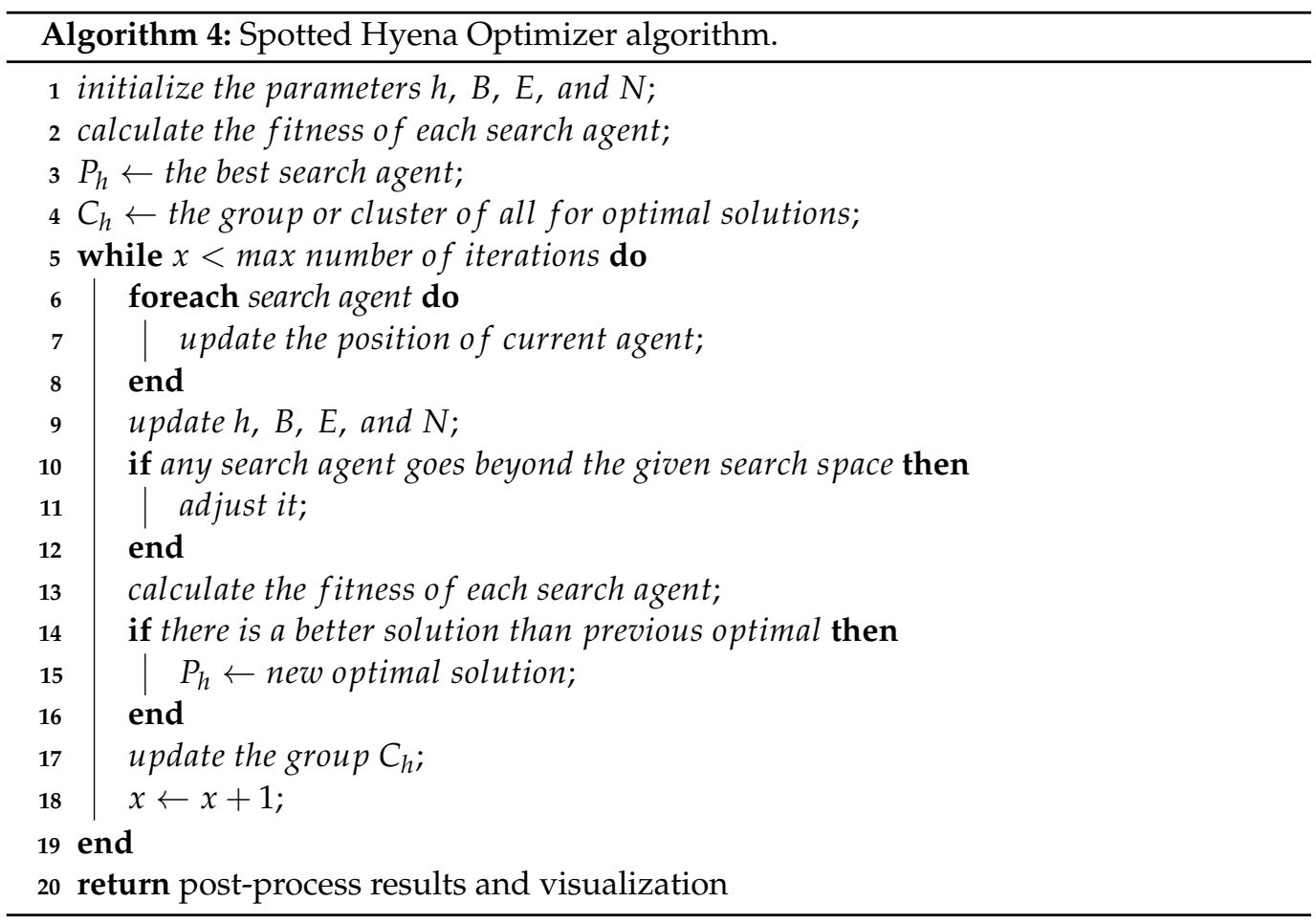

\section{Metodology}

\subsection{Platform}

The Amazon elastic cloud (EC2) platform was used as the experimental environment for both modalities, configuring two-processor machines with 8 GB of RAM memory. In case of the distributed version, an equivalent virtual machine was implemented for each node. Each of them was executed separately, and the parameter setting was determined experimentally for each instance group. They were selected according to Table 1.

The algorithms were written in Python 3.7, and the experiments were conducted for NR instances, proposed by the OR-Library [42] for the SCP (NRE to NRH). They instances consisted of datasets of $500 \times 5000$ and $1000 \times 10,000$ lines and columns, respectively, and they were unicost problems from [43]. The results obtained by standalone algorithms were taken as a basis for comparison to those that were subsequently generated with the distributed framework. The analysis was focused on this comparison, and not on improving results by tuning the parameters or modifying the operators. Once the standalone experiments were completed, the distributed experiments were run on the Spark 2.2.3, platform with the same parameters. As mentioned above, the execution was collective, i.e., all nodes participated in a coordinated way in the searching for best solutions, reporting results to the CC every 100 iterations. This value was determined experimentally, and it corresponds to the approximate average number of iterations in which the four algorithms show improvements in searching. If new algorithms are incorporated or proposed, or they are replaced, the value of this parameter should be reevaluated. This collaboration among different algorithms means that the optimum found is a consequence of the search in different neighborhoods, carried out by different algorithms. Therefore, it does not correspond to a single metaheuristic, even if it is a particular one that finds the best value. 
Table 1. Algorithms main parameters.

\begin{tabular}{cccccccc}
\hline BBH & \multicolumn{2}{c}{ SHO } & \multicolumn{2}{c}{ SLC } & \multicolumn{2}{c}{ SFLA } \\
\hline Parameter & Value & Parameter & Value & Parameter & Value & Parameter & Value \\
\hline Universe size & 70 & Hyenas quantity & 40 & Teams quantity & 10 & Memeplex quantity & 8 \\
Max iterations & 10,000 & Max iterations & 10,000 & Max iterations & 10,000 & Frogs in memeplex & 8 \\
& & & & Fixed players & 6 & Iter. memeplex & 30 \\
& & & Suplent players & 5 & Max iterations & 10,000 \\
& & & Im. factor & 5 & & \\
& & & Mult. factor & 5 & & \\
& & & Reduction & 15 & & \\
\end{tabular}

\subsection{Experimental Design}

Considering that the nodes of the distributed platform execute the same versions of the standalone algorithms, the improvement can be interpreted mainly as a product of two aspects of the proposed architecture: the clustering of the search space, and the collaboration among nodes. The resolution time and the number of calls to the objective function are variables subject to several conditions that make them not very objective. However, because the standalone and distributed experiments were run in similar environments and platforms, it is relevant to explain them.

The standalone experiments were performed by grouping by each of the instances and algorithms, i.e., 80 modalities that were run 31 times each, totaling 2480 experiments. In addition, the distributed version was run 31 times for each instance, i.e., 620 experiments.

Because the proposed method is not metaheuristic itself, but the coordinated work of several of them, it becomes relevant to figure out the contribution of each one in the complete search towards the optimum value. As noted above, every 100 iterations, the algorithms reported the best solution found to the CC. If this solution was better than the current optimal, the other algorithms were informed of the same, we can observe this behavior in the Figure 2.

If we consider the existing iterations since the previous optimum was received, and the new one that was found in an algorithm as collaboration time, we can quantify the percentage of participation for each algorithm in the search process. In the same way, it is important to know the behavior of this collaboration for different algorithms, in different stages of the search. For better analysis, the entire search process was divided into deciles.

To determine how decisive the number of nodes generated is in the results obtained, the experiments were replicated in three modalities. The first method consisted of generating $\mathrm{K}$ nodes, i.e., a unique node for each cluster. The second one consisted the generation of $2 \mathrm{~K}$ nodes. In other words, two nodes for each detected cluster. The third one, in analogy, corresponded to the generation of $3 \mathrm{~K}$ nodes.

Because $\mathrm{K}$ is generally larger than the number of used algorithms, for each round of experiments, the algorithms were assigned in fixed sequence, i.e., for $\mathrm{K}=4$, each cluster was addressed by a different algorithm, and for $\mathrm{k}>4$, the algorithms started repeating in a fixed order. 


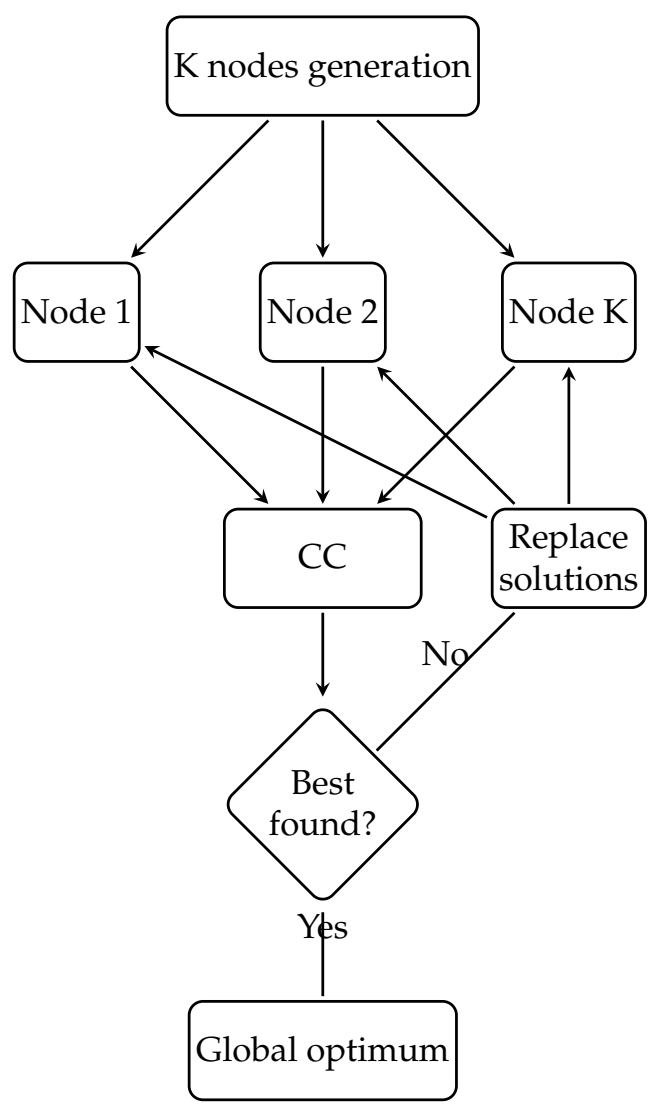

Figure 2. Procesing flow.

\section{Statistical Analysis}

Due to the stochastic nature of metaheuristics, it is not sufficient to present good results about the optimums found; it is important to demonstrate statistical regularity of the obtained values as well. This can be explained by results which do not necessarily satisfy qualities such as normality, homoscedasticity and independence, as demonstrated by [44,45]. For this purpose, Lanza and Gómez [46] proposed a methodology to compare two algorithms, or variations of them, considering the regularity and consistency of results. The details can be seen in the Figure 3:

The first step was to determine the outliers in the results of each algorithm, for all instances used. For this purpose, the average result for each of the experiments were calculated for every instance. The first and third quartiles and the interquartile range were calculated next. In the cases in which extreme outliers were found, these values were eliminated from the results table, leaving only the mild ones to be analyzed. The next steps were to determine the data distribution and independence, i.e., whether it has normal distribution or can be considered statistically belonging to different sets. For the first, the Shapiro-Wilk and Kolmogorov-Smirnov-Lilliefors tests were applied to detect the normality of the results. These tests were raised for the four algorithms used, and also for the distributed execution. To determine whether the proposed architecture was more regular and statistically coherent than each of the standalone algorithms, the Wilcoxon and Wilcoxon-Mann-Whitney tests were applied to the paired and independent datasets, respectively. This method was applied to each sequence of experiments in the three modalities described in the previous section. 


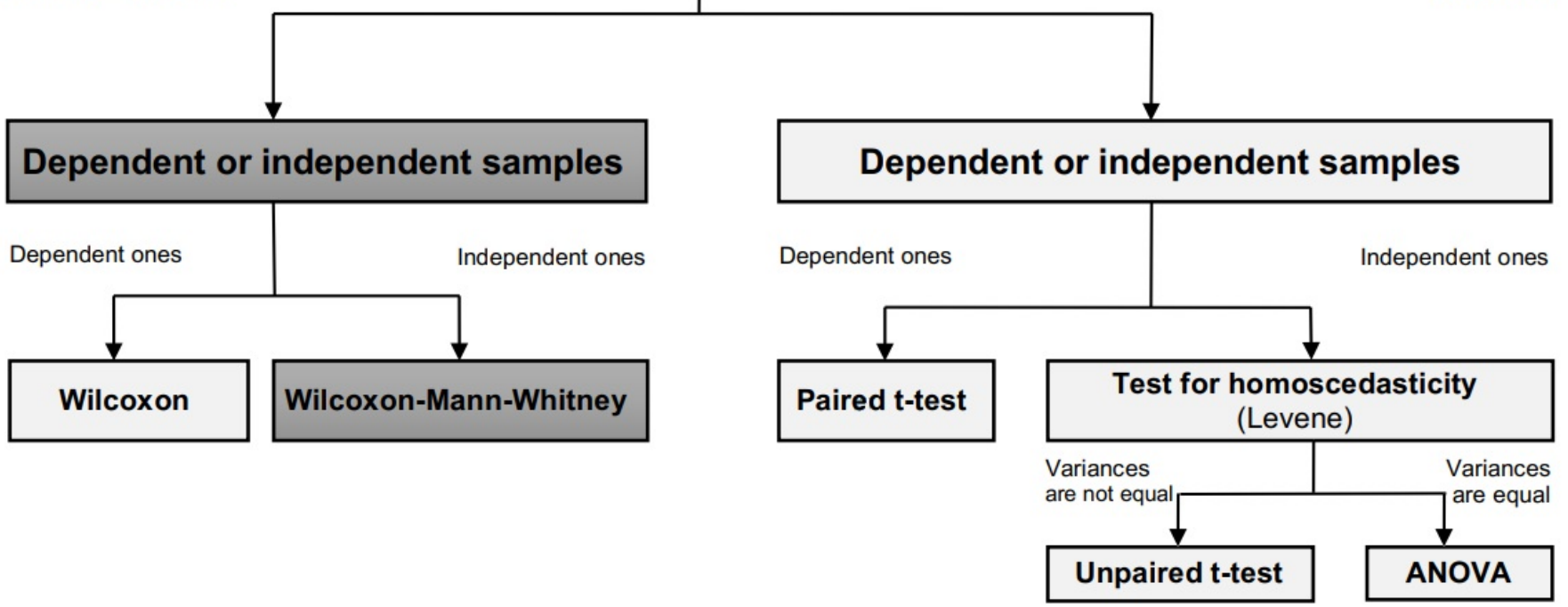

Figure 3. Statistical methodology.

\section{Experimental Results}

Table 2 shows the best results obtained for each instance of the set covering problem. These are highlighted in bold font. $Z_{B K S}$ is the known global optimum for each instance, while $\operatorname{Min}_{S F L A}, \mathrm{Min}_{S H O}, \mathrm{Min}_{S L C}$ and $\operatorname{Min}_{B B H}$ were the best values obtained for each instance by the standalone algorithms. The RPD in each experiment group is the relative percentage deviation from the known optimum, calculated as follows: $\left(\frac{\operatorname{Min}_{f o u n d} * 100}{Z_{b k s}}\right)-100$.

For the following tables, we report the $\operatorname{Min}_{D T}, \operatorname{Max}_{D T}, \operatorname{Med}_{D T}$ and $R P D_{D T}$ columns that show the minimum, maximum, median and RPD values obtained with the proposed framekork (DT).

Table 3 shows the best results obtained for each SCP benchmark by the version distributed on $\mathrm{K}$ nodes, compared to the RPDs with those of the standalone versions. Again, the best results are highlighted in bold font. The MFE corresponds to the number of evaluations performed of the objective function, and CT is the computation time in minutes for total execution.

Tables 4 and 5 show results for the execution with $2 \mathrm{~K}$ nodes and $3 \mathrm{~K}$ node, respectively. We employed bold font to illustrate the best results, again. In the resolved benchmarks, it is possible to notice a greater difference in the RPDs obtained, as well as in the number of found global optimums. This is relevant, since these instances represent types of problems that present difficulties in being solved using metaheuristics and demand higher computational power due to the large volume of data. 
Table 2. Results comparison for standalone versions.

\begin{tabular}{|c|c|c|c|c|c|c|c|c|c|}
\hline Instance & $Z_{B K S}$ & $\operatorname{Min}_{S F L A}$ & $R P D_{S F L A}$ & $\mathrm{Min}_{S H O}$ & $R P D_{S H O}$ & $\operatorname{Min}_{S L C}$ & $R P D_{S L C}$ & $\operatorname{Min}_{B B H}$ & $R P D_{B B H}$ \\
\hline NRE1 & 29 & 41 & 41.38 & 29 & 0.00 & 34 & 17.24 & 52 & 79.31 \\
\hline NRE2 & 30 & 31 & 3.33 & 32 & 6.67 & 37 & 23.33 & 46 & 53.33 \\
\hline NRE3 & 27 & 38 & 40.74 & 29 & 7.41 & 38 & 40.74 & 28 & 3.70 \\
\hline NRE4 & 28 & 40 & 42.86 & 32 & 14.29 & 31 & 10.71 & 29 & 3.57 \\
\hline NRE5 & 28 & 41 & 46.43 & 28 & 0.00 & 39 & 39.29 & 28 & 0.00 \\
\hline NRF1 & 14 & 14 & 0.00 & 18 & 28.57 & 23 & 64.29 & 17 & 21.43 \\
\hline NRF2 & 15 & 18 & 20.00 & 15 & 0.00 & 18 & 20.00 & 18 & 20.00 \\
\hline NRF3 & 14 & 16 & 14.29 & 14 & 0.00 & 20 & 42.86 & 14 & 0.00 \\
\hline NRF4 & 14 & 15 & 7.14 & 15 & 7.14 & 18 & 28.57 & 16 & 14.29 \\
\hline NRF5 & 13 & 16 & 23.08 & 14 & 7.69 & 17 & 30.77 & 14 & 7.69 \\
\hline NRG1 & 176 & 201 & 14.20 & 190 & 7.95 & 185 & 5.11 & 180 & 2.27 \\
\hline NRG2 & 151 & 166 & 9.93 & 158 & 4.64 & 176 & 16.56 & 153 & 1.32 \\
\hline NRG3 & 166 & 170 & 2.41 & 170 & 2.41 & 173 & 4.22 & 166 & 0.00 \\
\hline NRG4 & 168 & 169 & 0.60 & 170 & 1.19 & 181 & 7.74 & 197 & 17.26 \\
\hline NRG5 & 168 & 183 & 8.93 & 169 & 0.60 & 189 & 12.50 & 171 & 1.79 \\
\hline NRH1 & 63 & 68 & 7.94 & 66 & 4.76 & 69 & 9.52 & 71 & 12.70 \\
\hline NRH2 & 63 & 69 & 9.52 & 66 & 4.76 & 66 & 4.76 & 70 & 11.11 \\
\hline NRH3 & 59 & 63 & 6.78 & 59 & 0.00 & 66 & 11.86 & 67 & 13.56 \\
\hline NRH4 & 59 & 73 & 23.73 & 61 & 3.39 & 62 & 5.08 & 69 & 16.95 \\
\hline NRH5 & 55 & 70 & 27.27 & 58 & 5.45 & 61 & 10.91 & 64 & 16.36 \\
\hline
\end{tabular}

For the standalone versions of the SFLA, SHO, SLC and BBH algorithms, the average RPDs are 17.53, 5.35, 20.30 and 14.83, respectively, but for the distributed (DT) K, 2K and $3 \mathrm{~K}$ versions, they are $3.40,2.56$ and 2.10 , respectively, which are significantly lower. It is clear from the above tables that for the $\mathrm{K}, 2 \mathrm{~K}$ and $3 \mathrm{~K}$ node versions, $60 \%, 80 \%$ and $80 \%$, respectively, of the instances performed as well or better in the DT architecture than in any of the standalone algorithms. Figure 4 shows the average times in minutes used by the autonomous and DT algorithms that find the minimum values of some instances, or the output criteria, grouped by instance type. 
Table 3. K nodes RPD comparison for standalone and distributed versions.

\begin{tabular}{|c|c|c|c|c|c|c|c|c|c|c|c|}
\hline Instance & BKS & $M_{i n_{D T}}$ & $\operatorname{Max}_{D T}$ & $\operatorname{Med}_{D T}$ & $R P D_{D T}$ & $R P D_{S F L A}$ & $R P D_{S H O}$ & $R P D_{S L C}$ & $R P D_{B B H}$ & MFE & $\mathrm{CT}$ \\
\hline NRE1 & 29 & 29 & 55 & 43 & 0.00 & 41.38 & 0.00 & 17.24 & 79.31 & $1,071,926$ & 2.060 \\
\hline NRE2 & 30 & 32 & 58 & 49 & 6.67 & 3.33 & 6.67 & 23.33 & 53.33 & $1,068,618$ & 2.054 \\
\hline NRE3 & 27 & 28 & 33 & 30 & 3.70 & 40.74 & 7.41 & 40.74 & 3.70 & $1,000,391$ & 1.923 \\
\hline NRE4 & 28 & 29 & 56 & 46 & 3.57 & 42.86 & 14.29 & 10.71 & 3.57 & 922,880 & 1.774 \\
\hline NRE5 & 28 & 28 & 61 & 47 & 0.00 & 46.43 & 0.00 & 39.29 & 0.00 & 960,027 & 1845 \\
\hline NRF1 & 14 & 15 & 35 & 24 & 7.14 & 0.00 & 28.57 & 64.29 & 21.43 & $1,071,669$ & 1600 \\
\hline NRF2 & 15 & 15 & 29 & 23 & 0.00 & 20.00 & 0.00 & 20.00 & 20.00 & $1,158,639$ & 1729 \\
\hline NRF3 & 14 & 14 & 25 & 22 & 0.00 & 14.29 & 0.00 & 42.86 & 0.00 & $1,131,867$ & 1689 \\
\hline NRF4 & 14 & 15 & 22 & 19 & 7.14 & 7.14 & 7.14 & 28.57 & 14.29 & $1,225,920$ & 1830 \\
\hline NRF5 & 13 & 14 & 27 & 23 & 7.69 & 23.08 & 7.69 & 30.77 & 7.69 & $1,282,364$ & 1915 \\
\hline NRG1 & 176 & 178 & 219 & 195 & 1.14 & 14.20 & 7.95 & 5.11 & 2.27 & 876,533 & 2020 \\
\hline NRG2 & 151 & 153 & 190 & 172 & 1.32 & 9.93 & 4.64 & 16.56 & 1.32 & 952,975 & 2197 \\
\hline NRG3 & 166 & 172 & 199 & 182 & 3.61 & 2.41 & 2.41 & 4.22 & 0.00 & 966,945 & 2228 \\
\hline NRG4 & 168 & 174 & 205 & 191 & 3.57 & 0.60 & 1.19 & 7.74 & 17.26 & 897,329 & 2069 \\
\hline NRG5 & 168 & 175 & 226 & 201 & 4.17 & 8.93 & 0.60 & 12.50 & 1.79 & 929,964 & 2143 \\
\hline NRH1 & 63 & 66 & 83 & 75 & 4.76 & 7.94 & 4.76 & 9.52 & 12.70 & 968,004 & 1940 \\
\hline NRH2 & 63 & 65 & 85 & 74 & 3.17 & 9.52 & 4.76 & 4.76 & 11.11 & $1,052,336$ & 2109 \\
\hline NRH3 & 59 & 59 & 94 & 81 & 0.00 & 6.78 & 0.00 & 11.86 & 13.56 & $1,018,750$ & 2042 \\
\hline NRH4 & 59 & 63 & 100 & 83 & 6.78 & 23.73 & 3.39 & 5.08 & 16.95 & 989,600 & 1983 \\
\hline NRH5 & 55 & 57 & 101 & 77 & 3.64 & 27.27 & 5.45 & 10.91 & 16.36 & $1,050,947$ & 2.106 \\
\hline AVG & & & & & 3.40 & 17.53 & 5.35 & 20.30 & 14.83 & $1,029,884$ & 1963 \\
\hline
\end{tabular}

The above graphs show that the proposed architecture, on average, always solved the instances in less computational time than the best standalone algorithm.

Figure 5 shows how the average percentage of collaboration is distributed for each algorithm, considering all the resolved instances.

In Figure 6, the percentage of participation for each algorithm in each decile X-axis is represented on the Y-axis, so it is easy to observe the importance of each algorithm in all stages of the search. 
Table 4. 2K nodes RPD comparison for standalone and distributed versions.

\begin{tabular}{|c|c|c|c|c|c|c|c|c|c|c|c|}
\hline Instance & BKS & $\operatorname{Min}_{D T}$ & $\operatorname{Max}_{D T}$ & $\operatorname{Med}_{D T}$ & $R P D_{D T}$ & $R P D_{S F L A}$ & $R P D_{S H O}$ & $R P D_{S L C}$ & $R P D_{B B H}$ & MFE & CT \\
\hline NRE1 & 29 & 29 & 55 & 42 & 0.00 & 41.38 & 0.00 & 17.24 & 79.31 & $2,004,884$ & 2.493 \\
\hline NRE2 & 30 & 31 & 52 & 41 & 3.33 & 3.33 & 6.67 & 23.33 & 53.33 & $2,033,992$ & 2378 \\
\hline NRE3 & 27 & 28 & 33 & 30 & 3.70 & 40.74 & 7.41 & 40.74 & 3.70 & $1,999,607$ & 2490 \\
\hline NRE4 & 28 & 29 & 48 & 39 & 3.57 & 42.86 & 14.29 & 10.71 & 3.57 & $1,772,022$ & 1952 \\
\hline NRE5 & 28 & 28 & 44 & 36 & 0.00 & 46.43 & 0.00 & 39.29 & 0.00 & $1,908,427$ & 2156 \\
\hline NRF1 & 14 & 15 & 29 & 22 & 7.14 & 0.00 & 28.57 & 64.29 & 21.43 & $2,051,072$ & 1698 \\
\hline NRF2 & 15 & 15 & 30 & 22 & 0.00 & 20.00 & 0.00 & 20.00 & 20.00 & $2,137,033$ & 1657 \\
\hline NRF3 & 14 & 15 & 25 & 20 & 7.14 & 14.29 & 0.00 & 42.86 & 0.00 & $2,226,238$ & 1851 \\
\hline NRF4 & 14 & 14 & 21 & 18 & 0.00 & 7.14 & 7.14 & 28.57 & 14.29 & $2,299,324$ & 2301 \\
\hline NRF5 & 13 & 14 & 25 & 20 & 7.69 & 23.08 & 7.69 & 30.77 & 7.69 & $2,286,180$ & 2488 \\
\hline NRG1 & 176 & 177 & 201 & 189 & 0.57 & 14.20 & 7.95 & 5.11 & 2.27 & $1,597,704$ & 2497 \\
\hline NRG2 & 151 & 151 & 190 & 172 & 0.00 & 9.93 & 4.64 & 16.56 & 1.32 & $1,549,860$ & 2.517 \\
\hline NRG3 & 166 & 170 & 187 & 179 & 2.41 & 2.41 & 2.41 & 4.22 & 0.00 & $1,592,800$ & 3088 \\
\hline NRG4 & 168 & 171 & 176 & 174 & 1.79 & 0.60 & 1.19 & 7.74 & 17.26 & $1,586,827$ & 2515 \\
\hline NRG5 & 168 & 169 & 201 & 185 & 0.60 & 8.93 & 0.60 & 12.50 & 1.79 & $1,514,488$ & 2908 \\
\hline NRH1 & 63 & 65 & 83 & 74 & 3.17 & 7.94 & 4.76 & 9.52 & 12.70 & $1,901,904$ & 2324 \\
\hline NRH2 & 63 & 65 & 80 & 72 & 3.17 & 9.52 & 4.76 & 4.76 & 11.11 & $1,931,757$ & 2447 \\
\hline NRH3 & 59 & 59 & 79 & 69 & 0.00 & 6.78 & 0.00 & 11.86 & 13.56 & $1,867,105$ & 2615 \\
\hline NRH4 & 59 & 62 & 94 & 78 & 5.08 & 23.73 & 3.39 & 5.08 & 16.95 & $1,849,555$ & 2596 \\
\hline NRH5 & 55 & 56 & 77 & 67 & 1.82 & 27.27 & 5.45 & 10.91 & 16.36 & $1,922,298$ & 2.839 \\
\hline AVG & & & & & 2.56 & 17.53 & 5.35 & 20.30 & 14.83 & $1,901,654$ & 2391 \\
\hline
\end{tabular}

As it can be observed, the $\mathrm{SHO}$ and $\mathrm{BBH}$ algorithms participate in most of the optimum searches. The SFLA participates only partially, and the SLC only participates during the first iterations. One of the factors that explains this is the difference in the convergence rates of the algorithms. Those that converge faster have greater collaboration, but when the convergence rates of the algorithms are relatively similar, collaboration must be encouraged. This is the case for the SHO and BBH algorithms, which have similar convergence rates and cooperate with each other for most of the process, as opposed to the SLC, which has a very slow convergence. 

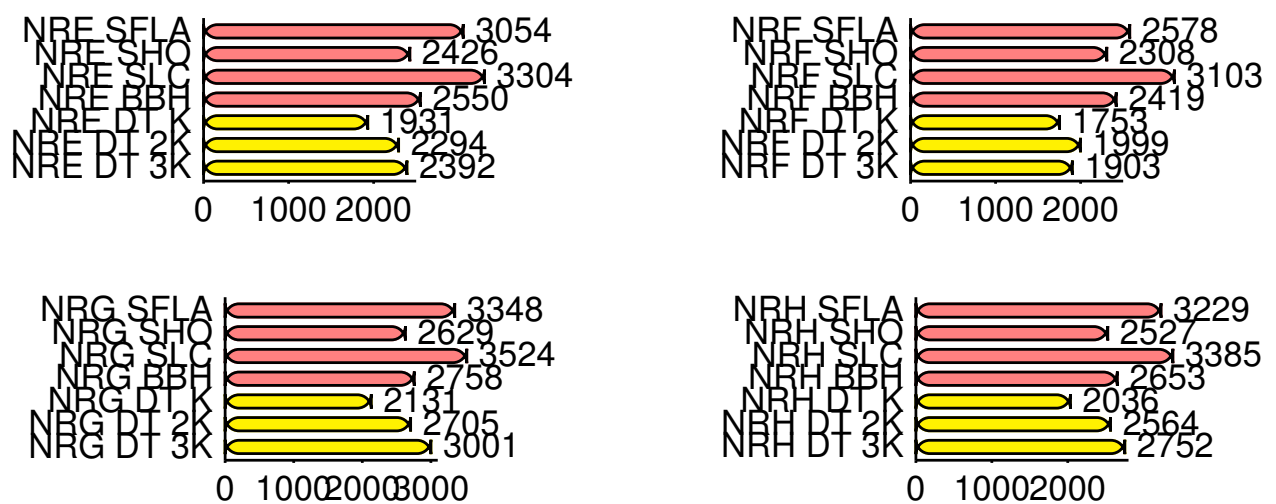

Figure 4. Average solving time comparison (mins).

Table 5. 3K nodes RPD comparison for standalone and distributed versions.

\begin{tabular}{|c|c|c|c|c|c|c|c|c|c|c|c|}
\hline Instance & BKS & $\operatorname{Min}_{D T}$ & $\operatorname{Max}_{D T}$ & $\operatorname{Med}_{D T}$ & $R P D_{D T}$ & $R P D_{S F L A}$ & $R P D_{S H O}$ & $R P D_{S L C}$ & $R P D_{B B H}$ & MFE & $\mathrm{CT}$ \\
\hline NRE1 & 29 & 29 & 52 & 41 & 0.00 & 41.38 & 0.00 & 17.24 & 79.31 & $2,909,407$ & 2.735 \\
\hline NRE2 & 30 & 31 & 52 & 42 & 3.33 & 3.33 & 6.67 & 23.33 & 53.33 & $2,827,970$ & 2640 \\
\hline NRE3 & 27 & 28 & 32 & 30 & 3.70 & 40.74 & 7.41 & 40.74 & 3.70 & $2,747,377$ & 2527 \\
\hline NRE4 & 28 & 29 & 48 & 39 & 3.57 & 42.86 & 14.29 & 10.71 & 3.57 & $2,589,137$ & 1900 \\
\hline NRE5 & 28 & 28 & 41 & 35 & 0.00 & 46.43 & 0.00 & 39.29 & 0.00 & $2,719,367$ & 2159 \\
\hline NRF1 & 14 & 15 & 29 & 22 & 7.14 & 0.00 & 28.57 & 64.29 & 21.43 & $2,899,910$ & 1449 \\
\hline NRF2 & 15 & 15 & 30 & 23 & 0.00 & 20.00 & 0.00 & 20.00 & 20.00 & $2,826,411$ & 1611 \\
\hline NRF3 & 14 & 14 & 22 & 18 & 0.00 & 14.29 & 0.00 & 42.86 & 0.00 & $2,904,276$ & 1697 \\
\hline NRF4 & 14 & 14 & 20 & 17 & 0.00 & 7.14 & 7.14 & 28.57 & 14.29 & $2,829,308$ & 2246 \\
\hline NRF5 & 13 & 14 & 25 & 20 & 7.69 & 23.08 & 7.69 & 30.77 & 7.69 & $2,734,454$ & 2514 \\
\hline NRG1 & 176 & 176 & 196 & 186 & 0.00 & 14.20 & 7.95 & 5.11 & 2.27 & $2,551,637$ & 2678 \\
\hline NRG2 & 151 & 154 & 188 & 171 & 1.99 & 9.93 & 4.64 & 16.56 & 1.32 & $2,645,503$ & 2958 \\
\hline NRG3 & 166 & 170 & 187 & 179 & 2.41 & 2.41 & 2.41 & 4.22 & 0.00 & $2,635,247$ & 3433 \\
\hline NRG4 & 168 & 171 & 176 & 174 & 1.79 & 0.60 & 1.19 & 7.74 & 17.26 & $2,688,812$ & 2764 \\
\hline NRG5 & 168 & 169 & 201 & 185 & 0.60 & 8.93 & 0.60 & 12.50 & 1.79 & $2,786,969$ & 3171 \\
\hline NRH1 & 63 & 65 & 80 & 73 & 3.17 & 7.94 & 4.76 & 9.52 & 12.70 & $2,891,291$ & 2428 \\
\hline NRH2 & 63 & 65 & 79 & 72 & 3.17 & 9.52 & 4.76 & 4.76 & 11.11 & $2,891,423$ & 2774 \\
\hline NRH3 & 59 & 59 & 77 & 68 & 0.00 & 6.78 & 0.00 & 11.86 & 13.56 & $2,946,120$ & 2799 \\
\hline NRH4 & 59 & 61 & 90 & 76 & 3.39 & 23.73 & 3.39 & 5.08 & 16.95 & $2,778,388$ & 2696 \\
\hline NRH5 & 55 & 55 & 76 & 66 & 0.00 & 27.27 & 5.45 & 10.91 & 16.36 & $3,001,766$ & 3063 \\
\hline AVG & & & & & 2.10 & 17.53 & 5.35 & 20.30 & 14.83 & $2,790,239$ & 2512 \\
\hline
\end{tabular}




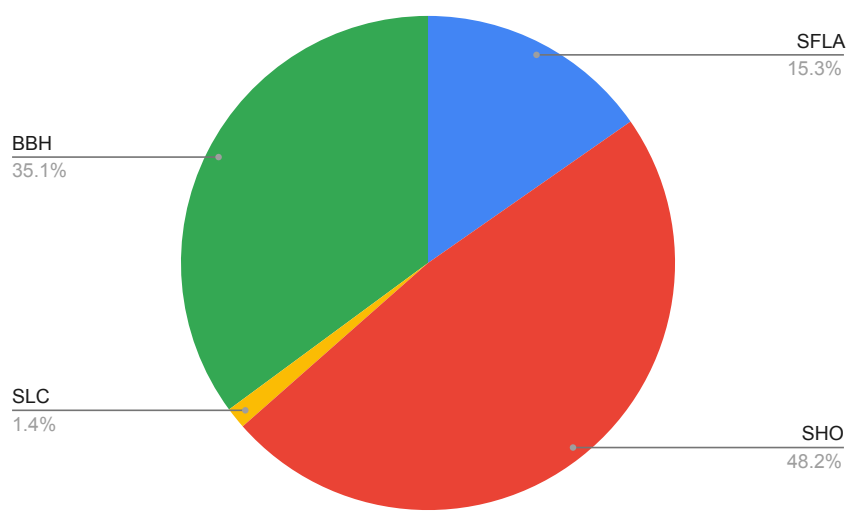

Figure 5. Average percentage of participation in the searching.

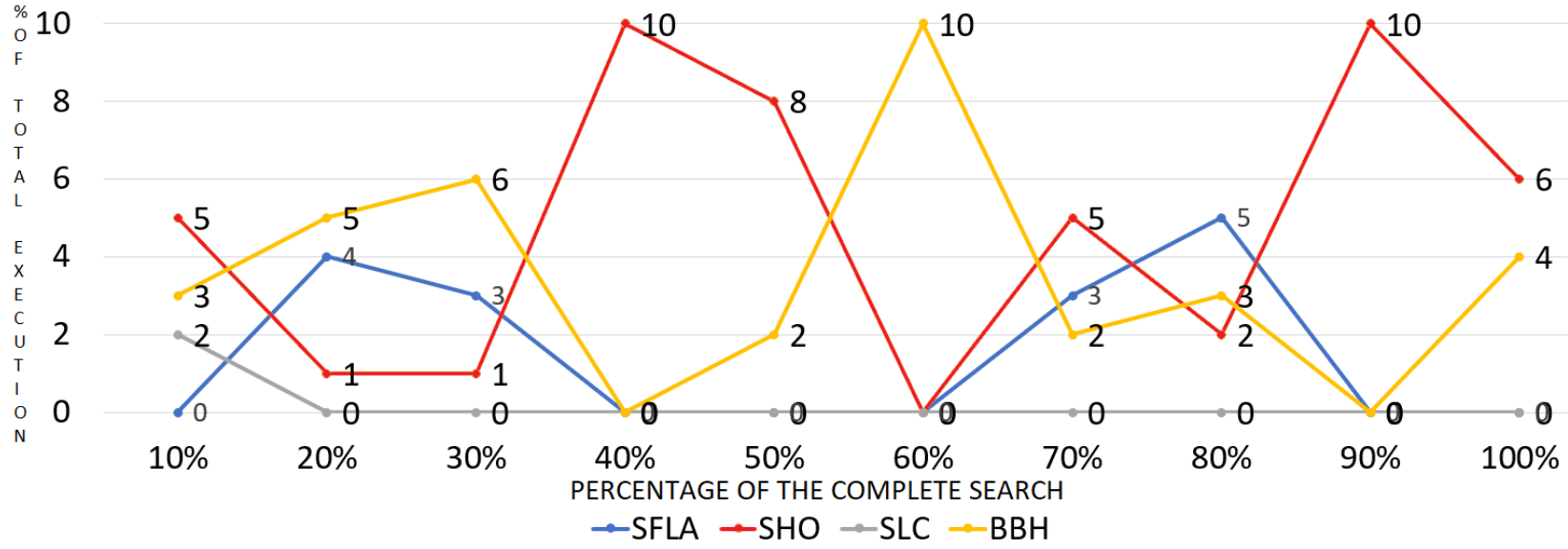

Figure 6. Collaboration evolution for each algorithm.

To show the different convergence rates of each algorithm and the DT version, Figure 7 plots the evolution of the minimums found in each iteration, for each algorithm, for one of the solved instances. The rest of the instances presented the same behavior, with small differences of low significance.

\section{Convergences best execution for HRF1 instance}

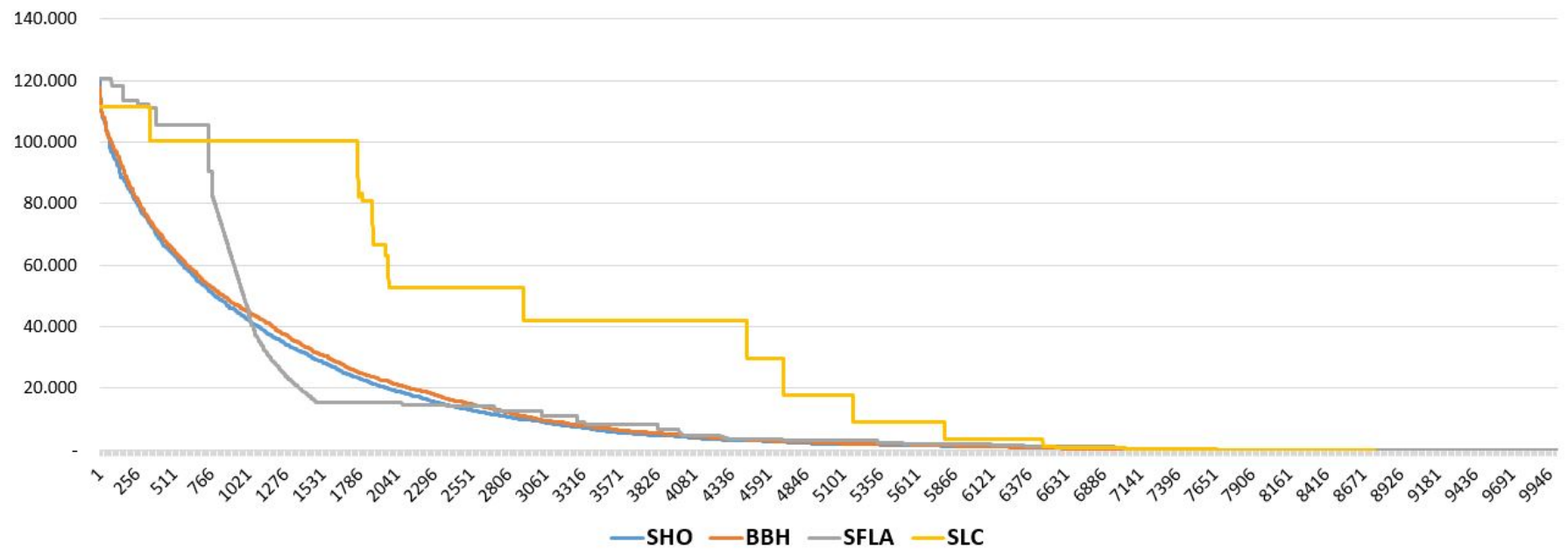

Figure 7. Convergences best execution of HRF1 instance. 


\section{Statistical Analysis}

With these results, a table was constructed to determine the outliers, according to the following conditions:

$$
\begin{aligned}
& \text { Mild outlier : value }<Q_{1}-(1.5 I Q R) \text { or }>Q_{3}+(1.5 I Q R) \\
& \text { Extreme outlier : value }<Q_{1}-(3 I Q R) \text { or }>Q_{3}+(3 I Q R)
\end{aligned}
$$

All the experiments had a $p$-value $<0.05$. Therefore, it cannot be assumed that H0 was satisfied for any of the instances or algorithms. Similarly, their non-normal distribution was validated, and the independence of the results obtained was tested, resulting in most of them being independent, although a few were classified as dependent. According to the methodology detailed above, it is necessary to determine the statistical consistency of the independent and DT algorithms by applying the Wilcoxon and Wilcoxon-Mann-Whitney tests to the paired and independent datasets, respectively. The analysis was performed with the DT with $\mathrm{K}, 2 \mathrm{~K}$ and $3 \mathrm{~K}$ nodes, but since the statistical results between them were not significantly different, we show the values obtained with K nodes. Table 6 shows the algorithm selected for all tested instances.

Figure 8 compares the global results distribution for statistical analysis. Al- most three quarters of instances have better statistical regularity in the distributed algorithm.

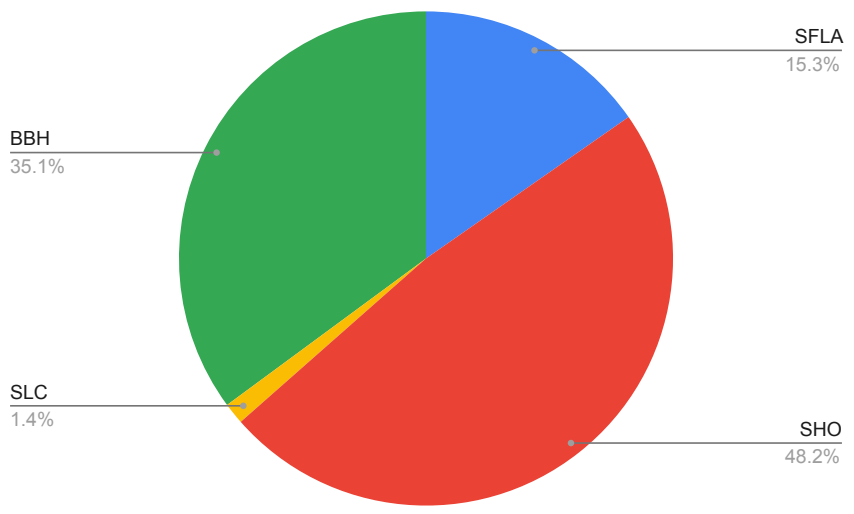

Figure 8. Algorithm selection distribution.

Table 7 compares the instances detailing the standalone and DT algorithms with the best resultsas well as the statistically selected ones. In those cases in which the first two concur, the respective line is noted (ND = no difference). We can observe that in $75 \%$ of the instances, there is a coincidence in the algorithm that reports the best result, regardless of which is statistically the best.

We can observe that in $75 \%$ of the instances there is a coincidence in the algorithm that reports the best result, regardless of which is selected as the best statistically.

Figures 9-12 show the results distribution for each instance, considering the minimum, maximum, median and interquartile distance for the DT algorithm version.

Some regularity can be observed in them, with a median approximately equidistant from the quartiles. The intervals between the maximum and minimum, as well as among the quartiles vary, according to the probabilistic nature of the algorithms. 
Table 6. Algorithm selected for tested instances.

\begin{tabular}{|c|c|c|c|c|c|c|c|}
\hline \multirow[b]{2}{*}{ Instance } & \multicolumn{2}{|c|}{ SFLA-DT } & \multicolumn{5}{|c|}{ SHO-DT } \\
\hline & Selected & Instance & Selected & Instance & Selected & Instance & Selected \\
\hline NRE1 & SFLA & NRG1 & DT & NRE1 & DT & NRG1 & DT \\
\hline NRE2 & DT & NRG2 & SFLA & NRE2 & $\mathrm{SHO}$ & NRG2 & $\mathrm{SHO}$ \\
\hline NRE3 & DT & NRG3 & DT & NRE3 & $\mathrm{SHO}$ & NRG3 & DT \\
\hline NRE4 & SFLA & NRG4 & ND & NRE4 & DT & NRG4 & ND \\
\hline NRE5 & $\mathrm{ND}$ & NRG5 & DT & NRE5 & DT & NRG5 & DT \\
\hline NRF1 & DT & NRH1 & DT & NRF1 & $\mathrm{SHO}$ & NRH1 & $\mathrm{SHO}$ \\
\hline NRF2 & DT & NRH2 & DT & NRF2 & $\mathrm{SHO}$ & NRH2 & DT \\
\hline NRF3 & DT & NRH3 & DT & NRF3 & DT & NRH3 & $\mathrm{SHO}$ \\
\hline NRF4 & DT & NRH4 & DT & NRF4 & DT & NRH4 & DT \\
\hline \multirow[t]{2}{*}{ NRF5 } & DT & NRH5 & DT & NRF5 & DT & NRH5 & DT \\
\hline & \multicolumn{2}{|c|}{ SCL-DT } & \multicolumn{5}{|c|}{ BBH-DT } \\
\hline Instance & Selected & Instance & Selected & Instance & Selected & Instance & Selected \\
\hline NRE1 & ND & NRG1 & DT & NRE1 & $\mathrm{BBH}$ & NRG1 & DT \\
\hline NRE2 & SLC & NRG2 & DT & NRE2 & DT & NRG2 & $\mathrm{DT}$ \\
\hline NRE3 & DT & NRG3 & DT & NRE3 & DT & NRG3 & DT \\
\hline NRE4 & SLC & NRG4 & DT & NRE4 & DT & NRG4 & DT \\
\hline NRE5 & DT & NRG5 & DT & NRE5 & DT & NRG5 & $\mathrm{DT}$ \\
\hline NRF1 & DT & NRH1 & DT & NRF1 & ND & NRH1 & DT \\
\hline NRF2 & DT & NRH2 & $\mathrm{ND}$ & NRF2 & DT & NRH2 & $\mathrm{BBH}$ \\
\hline NRF3 & DT & NRH3 & DT & NRF3 & DT & NRH3 & $\mathrm{BBH}$ \\
\hline NRF4 & DT & NRH4 & $\mathrm{DT}$ & NRF4 & $\mathrm{ND}$ & NRH4 & $\mathrm{BBH}$ \\
\hline NRF5 & DT & NRH5 & DT & NRF5 & DT & NRH5 & DT \\
\hline
\end{tabular}


Table 7. Results algorithm comparison.

\begin{tabular}{cccccccc}
\hline Instance & SA & DT & Selected & Instance & SA & DT & Selected \\
\hline NRE1 & SHO & SHO & SFLA & NRG1 & SHO & SHO & DT \\
\hline NRE2 & SFLA & SHO & DT & NRG2 & BBH & BBH & SFLA \\
\hline NRE3 & BBH & BBH & DT & NRG3 & SHO & SHO & DT \\
\hline NRE4 & SHO & SHO & SFLA & NRG4 & SHO & BBH & ND \\
\hline NRE5 & SHO & SHO & ND & NRG5 & SHO & SHO & DT \\
\hline NRF1 & SHO & BBH & DT & NRH1 & BBH & BBH & DT \\
\hline NRF2 & SHO & SHO & DT & NRH2 & SHO & SHO & DT \\
\hline NRF3 & BBH & BBH & DT & NRH3 & SHO & BBH & DT \\
\hline NRF4 & SHO & SHO & DT & NRH4 & SHO & SHO & DT \\
\hline NRF5 & SHO & SHO & DT & NRH5 & SHO & BBH & DT \\
\hline
\end{tabular}

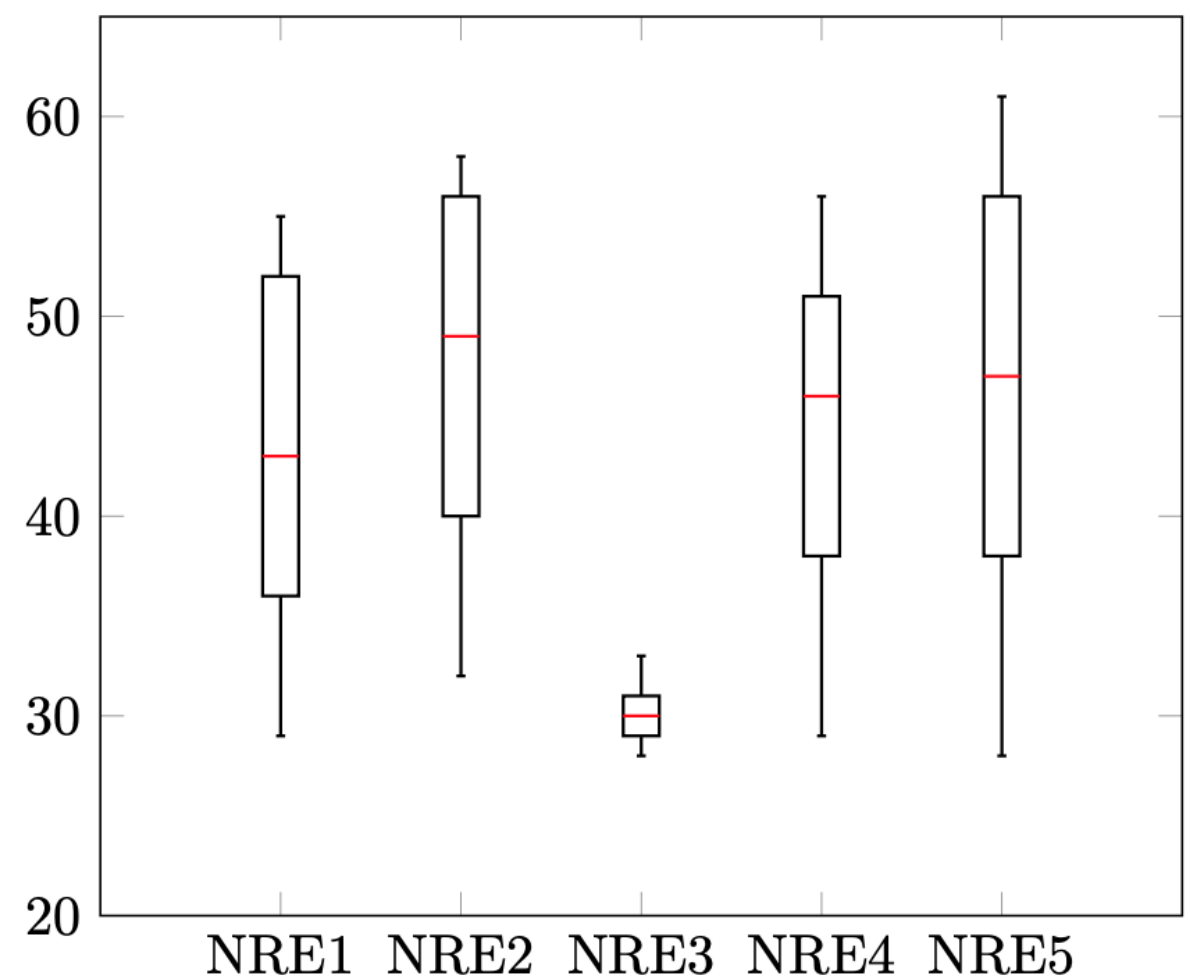

Figure 9. NRE Instances. 


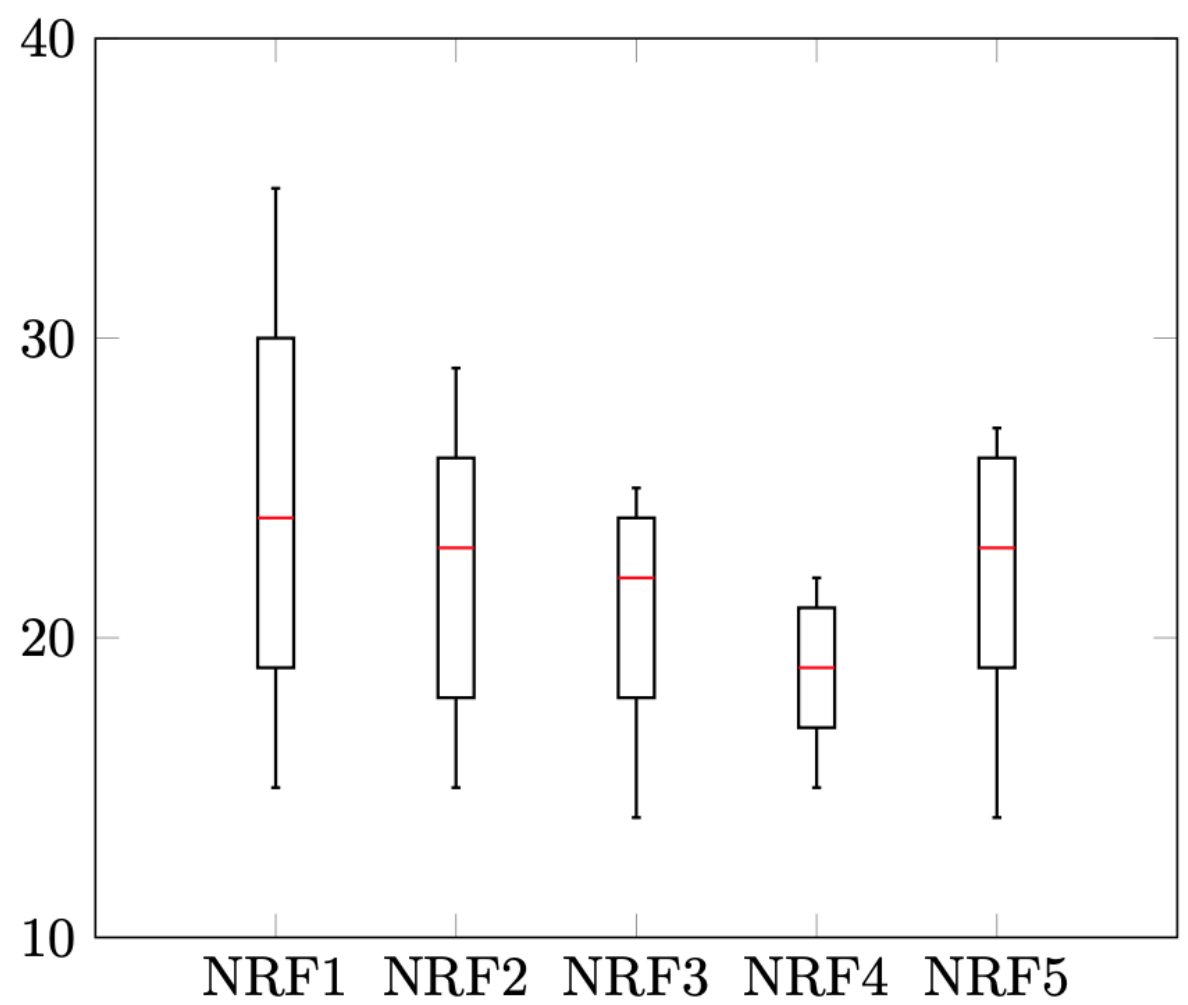

Figure 10. NRF Instances.

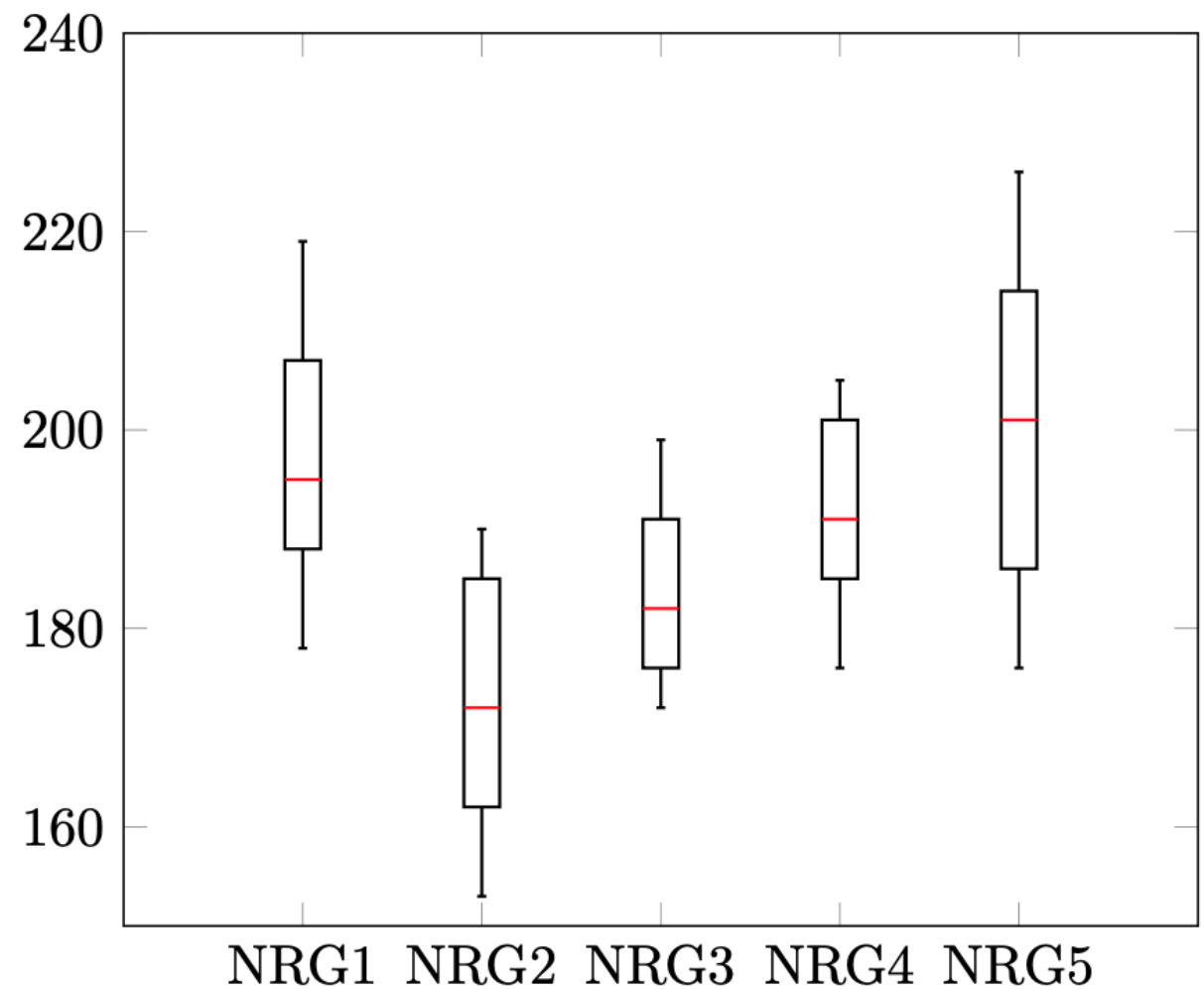

Figure 11. NRG Instances. 


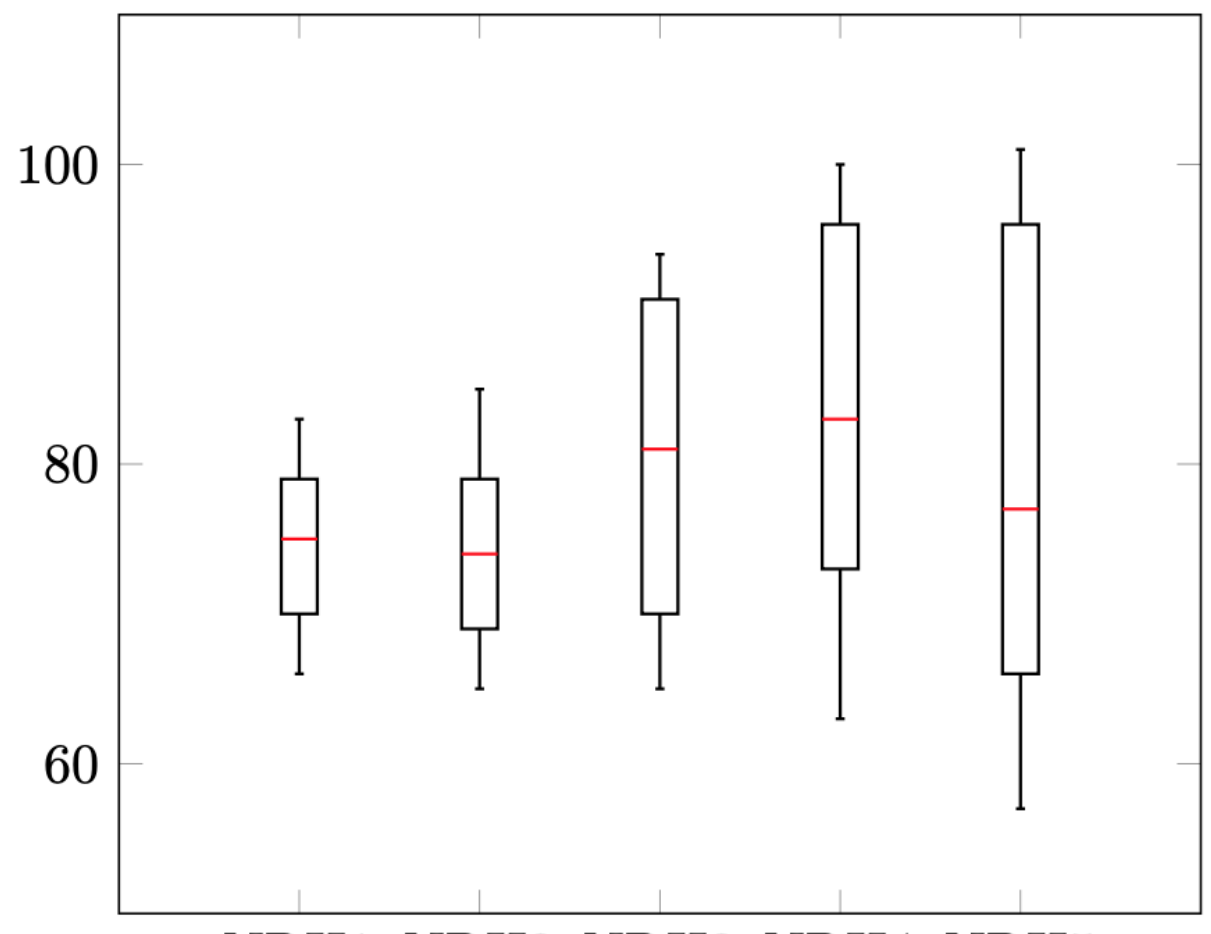

\section{NRH1 NRH2 NRH3 NRH4 NRH5}

Figure 12. NRH Instances.

\section{Conclusions}

In this work, we presented a framework that combines clustering and distributed parallelism to improve the metaheuristic search process. With the exception of a single instance, all of them showed improvements with regard to the best values obtained using the standalone algorithms or at least equaled them. In the experiments, $70 \%$ of the instances had the same or better RPD with the DT version than with the best of the standalone executions, achieving even more global optimums.

Removing the collaborative and clustering elements from the analysis results in a parallel execution of several algorithm instances processing the same dataset and with the same configuration, i.e., the equivalent of running the same number of experiments sequentially. We can then expect the results to be very similar to those obtained by the standalone experiments. However, this is not the case.

The results show an improvement in the RPD and statistical consistency of the DT executed algorithms. So, the key factors in any improvement are explained by the elements outside the algorithms, i.e., their collaborative work and the clustering of the search space. The obtained results reflect that the proposed architecture is more efficient when solving larger instances, making it a competitive alternative for difficult optimization problems.

From the results, we can see that although the number of nodes used is an important variable for improving the performance of a standalone algorithm, these improvements do not scale linearly, i.e., doubling the number of nodes does not necessarily double the number of best results, or halve the time required to find an optimum.

An important aspect is that the proposed approach, which is based on big data tools, makes solutions easier and cheaper, as it greatly automates the classic coding concerns associated with building distributed environments while maintaining an excellent degree of scalability. On the other hand, the clustering proved efficient in stimulating exploration of various types of neighborhoods, as the collaboration helped in exploiting those with good solutions. It should be noted that one aspect that appears to be important is that the correct selection of the algorithms to collaborate is crucial, thus making collaborations based on similar convergence rates a requisite. 
The results of this work suggest research that can allow greater control and predictability of the process, such as not only clustering the initial sets of solutions, but also during the process, as the results progress.

Author Contributions: Formal analysis, Á.G.-R., R.S., B.C. and R.O.; investigation, Á.G.-R., R.S., B.C., A.J., C.C. and R.O.; methodology, Á.G.-R., R.S. and C.C.; resources, R.S. and B.C.; software, Á.G.-R., A.J. and D.M.; validation, R.S., B.C., C.C. and R.O.; writing-original draft, Á.G.-R., A.J. and D.M.; writing-review \& editing, Á.G.-R., R.S., B.C., D.M., C.C. and R.O. All the authors of this paper hold responsibility for every part of this manuscript. All authors have read and agreed to the published version of the manuscript.

Funding: Ricardo Soto is supported by grant CONICYT/FONDECYT/REGULAR/1190129. Broderick Crawford is supported by grant ANID/FONDECYT/REGULAR/1210810. Álvaro Gómez-Rubio is supported by Postgraduate Grant Pontificia Universidad Católica de Valparaíso 2021.

Institutional Review Board Statement: Not applicable.

Informed Consent Statement: Not applicable.

Data Availability Statement: Not applicable.

Conflicts of Interest: The authors declare no conflict of interest. The founding sponsors had no role in the design of the study; in the collection, analyses, or interpretation of data; in the writing of the manuscript, and in the decision to publish the results.

\section{References}

1. Carbas, S.; Toktas, A.; Ustun, D. Nature-Inspired Metaheuristic Algorithms for Engineering Optimization Applications, 1st ed.; Springer: Berlin/Heidelberg, Germany, 2021.

2. Stegherr, H.; Heider, M.; Hähner, J. Classifying Metaheuristics: Towards a unified multi-level classification system. Nat. Comput. 2020. [CrossRef]

3. Morales-Castañeda, B.; Zaldívar, D.; Cuevas, E.; Fausto, F.; Rodríguez, A. A better balance in metaheuristic algorithms: Does it exist? Swarm Evol. Comput. 2020, 54, 100671. [CrossRef]

4. Hatamlou, A. Black hole: A new heuristic optimization approach for data clustering. Inf. Sci. 2013, 222, 175-184. [CrossRef]

5. Jaramillo, A.; Crawford, B.; Soto, R.; Mansilla, S.; Rubio, Á.G.; Salas, J.; Olguín, E. Solving the Set Covering Problem with the Soccer League Competition Algorithm. Trends Appl. Knowl.-Based Syst. Data Sci. 2014, 9799, 884-891. [CrossRef]

6. Eusuff, M.; Lansey, K.; Pasha, F. Shuffled frog-leaping algorithm: A memetic meta-heuristic for discrete optimization. Eng. Optim. 2004, 9799, 884-891. [CrossRef]

7. Dhiman, G.; Kumar, V. Spotted hyena optimizer: A novel bio-inspired based metaheuristic technique for engineering applications. Adv. Eng. Softw. 2017, 114, 48-70. [CrossRef]

8. García, J.; Yepes, V.; Martí, J. A Hybrid k-Means Cuckoo Search Algorithm Applied to the Counterfort Retaining Walls Problem. Mathematics 2020, 8, 555. [CrossRef]

9. Cheng, S.; Liu, B.; Ting, T.; Qin, Q.; Shi, Y.; Huang, K. Survey on data science with population-based algorithms. Big Data Anal. 2016, 1, 3. [CrossRef]

10. Harifi, S.; Khalilian, M.; Mohammadzadeh, J.; Ebrahimnejad, S. Using Metaheuristic Algorithms to Improve k-Means Clustering: A Comparative Study. Rev. D'Intell. Artif. 2020, 34, 297-305. [CrossRef]

11. Olivares, R.; Muñoz, R.; Soto, R.; Crawford, B.; Cárdenas, D.; Ponce, A.; Taramasco, C. An Optimized Brain-Based Algorithm for Classifying Parkinson's Disease. Appl. Sci. 2020, 10, 1827. [CrossRef]

12. Hardi, M.; Zrar, A.; Tarik, R.; Abeer, A.; Nebojsa, B. A new K-means grey wolf algorithm for engineering problems. World J. Eng. 2021, 18, 630-638. [CrossRef]

13. Muñoz, R.; Olivares, R.; Taramasco, C.; Villarroel, R.; Soto, R.; Barcelos, T.; Merino, E.; Alonso-Sánchez, M.F. Using Black Hole Algorithm to Improve EEG-Based Emotion Recognition. Comput. Intell. Neurosci. 2018, 2018, 3050214. [CrossRef] [PubMed]

14. SaiSindhuTheja, R.; Shyama, G. An efficient metaheuristic algorithm based feature selection and recurrent neural network for DoS attack detection in cloud computing environment. Appl. Soft Comput. 2021, 100, 106997. [CrossRef]

15. Bacanin, N.; Bezdan, T.; Tuba, E.; Strumberger, I.; Tuba, M. Optimizing Convolutional Neural Network Hyperparameters by Enhanced Swarm Intelligence Metaheuristics. Algorithms 2020, 13, 67. [CrossRef]

16. Santamaría, J.; Rivero-Cejudo, M.; Martos-Fernández, M.; Roca, F. An Overview on the Latest Nature-Inspired and MetaheuristicsBased Image Registration Algorithms. Appl. Sci. 2020, 10, 1928. [CrossRef]

17. Oliveira, G.; Coutinho, F.; Campellob, R.; Naldi, M. Improving k-means through distributed scalable metaheuristics. Neurocomputing 2017, 246, 45-57. [CrossRef]

18. Zhang, P.; Yin, Z.Y.; Jin, Y.F.; Chan, T.; Gao, F.P. Intelligent modelling of clay compressibility using hybrid meta-heuristic and machine learning algorithms. Geosci. Front. 2021, 12, 441-452. [CrossRef] 
19. Mallick, J.; Alqadhi, S.; Talukdar, S.; AlSubih, M.; Ahmed, M.; Abad, R.; Ben, N.; Abutayeh, S. Risk Assessment of Resources Exposed to Rainfall Induced Landslide with the Development of GIS and RS Based Ensemble Metaheuristic Machine Learning Algorithms. Sustainability 2021, 13, 457. [CrossRef]

20. Nayak, J.; Naik, B.; Byomakesha, P.; Pelusi, D. Optimal Fuzzy Cluster Partitioning by Crow Search Meta-Heuristic for Biomedical Data Analysis. Int. J. Appl. Metaheuristic Comput. 2021, 12, 49-66. [CrossRef]

21. Memeti, S.; Pllana, S.; Binotto, A.; Kołodziej, J.; Brandic, I. Using meta-heuristics and machine learning for software optimization of parallel computing systems: A systematic literature review. Computing 2019, 101, 893-936. [CrossRef]

22. Talbi, E.G. Machine Learning into Metaheuristics: A Survey and Taxonomy. ACM Comput. Surv. 2022, 54, 1-32. [CrossRef]

23. Hassan, A.; Pillay, N. Hybrid metaheuristics: An automated approach. Expert Syst. Appl. 2019, 130, 132-144. [CrossRef]

24. Oliva, D.; Hinojosa, S. Applications of Hybrid Metaheuristic Algorithms for Image Processing, 1st ed.; Springer: Berlin/Heidelberg, Germany, 2020.

25. Pellerin, R.; Perrier, N.; Berthaut, F. A survey of hybrid metaheuristics for the resource-constrained project scheduling problem. Eur. J. Oper. Res. 2020, 280, 395-416. [CrossRef]

26. Shukla, A.K.; Singh, P.; Vardhan, M. Gene selection for cancer types classification using novel hybrid metaheuristics approach. Swarm Evol. Comput. 2020, 54, 100661. [CrossRef]

27. Fakhrzad, M.B.; Goodarzian, F.; Golmohammadi, A.M. Addressing a fixed charge transportation problem with multi-route and different capacities by novel hybrid meta-heuristics. J. Ind. Syst. Eng. 2019, 12, 167-184.

28. Ikeda, S.; Nagai, T. A novel optimization method combining metaheuristics and machine learning for daily optimal operations in building energy and storage systems. Appl. Energy 2021, 289, 116716. [CrossRef]

29. Hijazi, N.; Faris, H.; Aljarah, I. A parallel metaheuristic approach for ensemble feature selection based on multi-core architectures. Expert Syst. Appl. 2021, 182, 115290. [CrossRef]

30. Arkhipov, D.; Wu, D.; Wu, T.; Regan, A. A Parallel Genetic Algorithm Framework for Transportation Planning and Logistics Management. Intell. Logist. Based Big Data 2020, 8, 106506-106515. [CrossRef]

31. Khanduja, N.; Bhushan, B. Recent Advances and Application of Metaheuristic Algorithms: A Survey (2014-2020). Metaheuristic Evol. Comput. Algorithms Appl. 2020, 916, 207-228. [CrossRef]

32. Mouton, J.; Ferreira, M.; Helberg, A. A comparison of clustering algorithms for automatic modulation classification. Expert Syst. Appl. 2020, 151, 113-317. [CrossRef]

33. Moosavian, N.; Kasaee, B. Algorithm AS 136: A K-Means Clustering Algorithm. J. R. Stat. Soc. Ser. C 2014, 17, 14-24.

34. Gowda, K.C.; Krishna, G. Agglomerative clustering using the concept of mutual nearest neighbourhood. Pattern Recognit. 1978, 10, 105-112. [CrossRef]

35. Sridevi, K.; Prakasha, S. Comparative Study on Various Clustering Algorithms Review. In Proceedings of the 5th International Conference on Intelligent Computing and Control Systems (ICICCS), Madurai, India, 6-8 May 2021; Volume 151, pp. 153-158. [CrossRef]

36. Zhou, H.; Gao, J. Automatic Method for Determining Cluster Number Based on Silhouette Coefficient. Adv. Mater. Res. 2014, 951, 227-230. [CrossRef]

37. Karp, R. Reducibility Among Combinatorial Problems. 1972. Available online: https://cs.stanford.edu/people/trevisan/cs172-0 4/karp.pdf (accessed on 13 January 2021).

38. Daskin, M. Application of an expected covering model to emergency medical service system design. Decis. Sci. 1982, 13, 416-439. [CrossRef]

39. Chen, L.; Yuan, D. Solving a minimum-power covering problem with overlap constraint for cellular network design. Eur. J. Oper. Res. 2010, 203, 714-723. [CrossRef]

40. ReVelle, C.; Toregas, C.; Falkson, L. Applications of the Location Set-covering Problem. Geogr. Anal. 1976, 8, 65-76. [CrossRef]

41. Moosavian, N.; Kasaee, B. Soccer league competition algorithm: A novel meta-heuristic algorithm for optimal design of water distribution networks. Swarm Evol. Comput. 2014, 17, 14-24. [CrossRef]

42. Beasley, J. OR-Library, 1990. Available online: http:// people.brunel.ac.uk/ mastjjb/jeb/info.html (accessed on 13 January 2021).

43. Grossman, T.; Wool, A. Computational experience with approximation algorithms for the set covering problem. Eur. J. Oper. Res. 1997, 101, 81-92. [CrossRef]

44. Derrac, J.; García, S.; Molina, D.; Herrera, F. A practical tutorial on the use of nonparametric statistical tests as a methodology for comparing evolutionary and swarm intelligence algorithms. Swarm Evol. Comput. 2011, 1, 3-18. [CrossRef]

45. García, S.; Molina, D.; Lozano, M.; Herrera, F. A study on the use of non-parametric tests for analyzing the evolutionary algorithms behaviour: A case study onthecec2005 special session onreal parameter optimization. J. Heuristics 2008, 15, 617-644. [CrossRef]

46. Lanza-Gutierrez, J.; Gómez-Pulido, J. Assuming multiobjective metaheuristics to solve a three-objective optimization problem for relay node deployment in wireless sensor networks. Appl. Soft Comput. 2015, 30, 675-687. [CrossRef] 